\title{
Lowest order in inelastic tunneling approximation: Efficient scheme for simulation of inelastic electron tunneling data
}

\author{
E. T. R. Rossen* and C. F. J. Flipse ${ }^{\dagger}$ \\ Department of Applied Physics, Eindhoven University of Technology, P.O. Box 513, 5600 MB, Eindhoven, The Netherlands \\ J. I. Cerdá \\ Instituto de Ciencia de Materiales de Madrid, ICMM-CSIC, Cantoblanco, 28049 Madrid, Spain \\ (Received 18 February 2013; revised manuscript received 28 May 2013; published 12 June 2013)
}

\begin{abstract}
We have developed an efficient and accurate formalism which allows the simulation at the $a b$ initio level of inelastic electron tunneling spectroscopy data under a scanning tunneling microscope setup. It exploits fully the tunneling regime by carrying out the structural optimization and vibrational mode calculations for surface and tip independently. The most relevant interactions in the inelastic current are identified as the inelastic tunneling terms, which are taken into account up to lowest order, while all other inelastic contributions are neglected. As long as the system is under tunneling regime conditions and there is no physisorbed molecule on the surface or tip apex, this lowest order in inelastic tunneling (LOIT) approach reduces drastically the computational cost compared to related approaches while maintaining a good accuracy. Adopting the wide-band limit for both tip and surface further reduces calculation times significantly, and is shown to give similar results to when the full energy dependence of the Green's functions is taken into account. The LOIT is applied to the $\mathrm{Cu}(111)+\mathrm{CO}$ system probed by a clean and a CO contaminated tip to find good agreement with previous works. Different parameters involved in the calculations such as basis sets, $k$ sampling, tip-sample distance, or temperature, among others, are discussed in detail.
\end{abstract}

DOI: 10.1103/PhysRevB.87.235412

PACS number(s): 68.43.Pq, 68.35.Ja, 68.37.Ef, 73.40.Gk

\section{INTRODUCTION}

The electronic transport across single molecules has become an active research field especially during the last years due to its implications on the still promising molecular electronics technology. ${ }^{1,2}$ There exist several experimental techniques that allow us to measure current versus voltage spectra $[I(V)$ curves $]$ in an electrode-molecule-electrode device, such as embedding the molecules between crosswires $^{3}$ or within a mechanical break junction ${ }^{4,5}$ or employing the scanning tunneling microscope (STM) setup whereby molecules are deposited on a metallic surface and contacted by the tip apex. Among them, the STM possesses the unique feature of first allowing an accurate localization of the molecule via topographic imaging and next measure its $I(V)$ characteristics as a function of the tip-sample relative position. Analyzing the second derivative $\partial^{2} I / \partial V^{2}$ yields relevant information on the molecular vibrations, ${ }^{6-8}$ as peak/dips in the spectra may be associated to inelastic processes involving the absorption or emission of vibrational modes (VMs). Within the context of the STM, this technique is denoted as inelastic electron tunneling spectroscopy (STM-IETS) and is extensively employed to characterize the molecule's vibrational spectra. ${ }^{9-15}$

From the theoretical side, a wealth of formalisms dealing with the inelastic transport have been developed in parallel to these delicate experiments. ${ }^{16-30}$ The earliest models for IETS employed a multiple scattering formalism (multichannels) with simplified semiempirical Hamiltonians ${ }^{16}$ or a Tersoff-Hamman-(TH-) type approach. ${ }^{17}$ More recently, most formalisms have been developed under the Born approximation (BA), either in its self-consistent version ${ }^{20-22}$ (SCBA) or perturbatively, typically exploiting the small $e$-VM coupling and/or the small applied bias. ${ }^{20,23,26}$ Whereas the SCBA requires large computational resources and therefore its use is generally limited within simple one-dimensional (1D) effective models, perturbative approaches permit lighter implementations and the use of $a b$ initio schemes for the evaluation of the Hamiltonian terms. Apart from TH-based approaches, ${ }^{17-19,29}$ which are specifically suited for the STM setup albeit they neglect any tip features, it is surprising that hardly any of the above-mentioned schemes, when applied to the simulation of IETS spectra, take additional advantage of the small tunneling terms typically involved in STM-IETS experiments. An exception is the work of Teobaldi et al., ${ }^{30}$ who under the assumptions of weak tip-sample interactions implemented a plane-wave Bardeen-type formalism for the inelastic currents explicitly including the tip structure.

In this work, we revisit the BA fully exploiting the tunneling regime conditions (TRCs) in order to derive an efficient and reliable formalism suited for the simulation of STM-IETS spectra. Our approach is based on the so-called lowest order expansion (LOE) of Paulsson and co-workers, ${ }^{26}$ which grasps the leading terms of the BA and by further assuming flat electronic bands around the Fermi level it becomes a computationally very efficient, yet accurate, form of calculating IETS. However, the LOE considers the entire STM interface as a single block attached to the electrodes and, hence, does not take advantage of the TRCs. In our case, and as sketeched in Fig. 1, we split the interface into two blocks, the surface and the tip apex, and generalize the SCBA equations for such system. Upon assuming weak $e$-VM coupling, weak surfacetip interactions, and a small applied bias, we arrive at a lowest order in inelastic tunneling (LOIT) approximation which consists of retaining only those terms coupling the tunneling electron with a VM located either at the surface or the tip and neglecting any inelastic events within each block. The accuracy of the LOIT is found excellent as long as the molecules are strongly binded (chemisorbed) to the surface or tip. 
The formalism has been implemented within the GREEN (Refs. 31 and 32) package, which is specially designed for the modelization of the complex STM interface. For instance, the apex is described as a nonperiodic cluster stacked below the tip electrode which may differ in nature from the sample electrode. All Hamiltonian terms are evaluated in a localized atomic orbital (AO) basis at the $a b$ initio level via an interface to the density functional theory (DFT) SIESTA (Ref. 33) code. The simplifications brought in by the LOIT approximation represent a considerable reduction in the computational cost and allow the use of highly extended AOs which provide a more accurate description of the electronic wave functions in the tunneling region as well as to consider independent $k$ samplings at the surface and tip. ${ }^{31}$ It also enables a particularly simple derivation of the IETS rules which govern the inelastic activity of a given mode. ${ }^{34}$

The paper is organized as follows. In Sec. II, we deduce the LOIT equations and prove its accuracy for an effective one-dimensional (1D) model. Section III describes the details of the LOIT implementation for realistic three-dimensional (3D) models, while in Sec. IV we present an analysis of the different parameters entering the IETS simulations. In Sec. V, the formalism is applied to the $\mathrm{Cu}(111)+\mathrm{CO}$ system probed by a clean and a $\mathrm{CO}$ functionalized tip, also addressing the fulfillment of the IETS selection rules and the dependence of the peak/dip heights on the tip-sample distance. The last section includes some final remarks and the conclusions.

\section{1D MODEL}

\section{A. Two-level system}

Let us first focus on a simple one-dimensional (1D) model for the STM system. In Fig. 1(a) we sketch the widely used onelevel system (1LS) consisting of a single impurity $i$ embedded between two electrodes. The left electrode $L$ corresponds to the sample, the right electrode $R$ to the STM tip, and $i$ refers to a molecule or atom adsorbed on the surface. The electrodes act as electronic reservoirs emitting electrons in thermal equilibrium with chemical potentials $\mu_{L / R}$. The coupling of the impurity to the left and right electrodes is described by the $H_{i L}$ and $H_{i R}$ interactions, respectively. Under the tunneling regime, the tip will be some $\AA$ away from the molecule and one may assume $H_{i R} / H_{i L} \ll 1$. Furthermore, all $e$-VM couplings are confined within the impurity and will be denoted by $M_{i i}^{\lambda}$, with $\lambda$ labeling each VM. Such a system has been solved in various works, often at the SCBA level, in order to establish the origin of peaks and dips in the IETS spectra. ${ }^{21,22,25}$ (a)

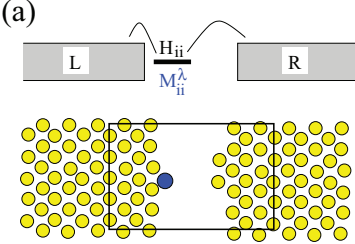

(b)

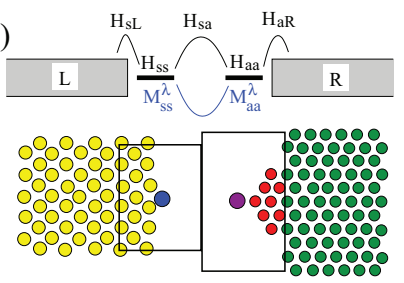

FIG. 1. (Color online) Sketch of a 1D model for the STM system assuming (a) a one-level system (1LS) and (b) a two-level system (2LS). The associated 3D models are drawn below. Rectangles enclose the atomistic generalization of each level.
However, the 1LS is not particularly well suited for the calculation and interpretation of STM-IETS data. First, the Green's function projection at the impurity is calculated without taking advantage of the weak molecule-tip $\left(H_{i R}\right)$ interaction and, second, inelastic processes are only considered within the molecule disregarding any inelastic tunneling events. For realistic calculations [bottom sketch in Fig. 1(a)], they are actually taken into account but at a large computational cost.

These two shortcomings can be addressed by extending the 1LS to a two-level system (2LS) as depicted in Fig. 1(b). Here, the single impurity is replaced by two levels denoted by $s$ and $a$. The former represents the molecule adsorbed at the surface, while the latter represents the tip apex, which may either be a metallic atom or a molecule/atom attached to the tip electrode. The vacuum gap responsible for the tunneling corresponds to the region between the two molecules and, therefore, the molecule-electrode interactions $H_{s L}$ and $H_{a R}$ will be much larger than the molecule-molecule interaction $H_{s a}\left(H_{s a} / H_{s L / a R} \ll 1\right)$. We allow for inelastic process within either molecule $M_{s s}^{\lambda}$ and $M_{a a}^{\lambda}$, and most importantly, we also consider explicitly inelastic tunneling via $M_{s a}^{\lambda}$.

Neglecting any Coulomb blockade effects and after applying an external bias $V$ between both electrodes, the current $I(V)$ across the STM junction will reach a steady state, in which case it becomes independent of the position in the chain and may be expressed as:

$$
I(V)=\frac{2 e}{h} \int_{-\infty}^{+\infty} g_{\alpha}(E, V) d E,
$$

where $g_{\alpha}(E, V)$ is the out-of-equilibrium differential current which may be calculated at either molecule $\alpha=s$ or $a$. We will assume throughout this work spin degeneracy, although the generalization to spin-polarized systems is straightforward.

A general form for $g_{\alpha}(E, V)$ is provided by the MeirWingreen formula

$$
g_{\alpha}(E, V)=\operatorname{Tr}\left[\Sigma_{\alpha \alpha}^{<, \gamma} G_{\alpha \alpha}^{>, \mathrm{ph}}-\Sigma_{\alpha \alpha}^{>, \gamma} G_{\alpha \alpha}^{<, \mathrm{ph}}\right],
$$

where $G_{\alpha \alpha}^{\gtrless, \text { ph }}$ corresponds to the greater and lesser Green's functions of the complete chain dressed by the vibrational modes and projected onto level $\alpha$ and $\Sigma_{\alpha \alpha}^{\gtrless, \gamma}$ stands for the greater and lesser self-energies arising from the electrode to which $\alpha$ is attached, i.e., $\gamma=L(R)$ if $\alpha=s(a)$.

Solving Eq. (2) is a rather complex problem since both the potential drop across the junction and the $e$-VM couplings should be solved self-consistently. However, under tunneling regime conditions (TRCs) we may assume that each molecule remains in equilibrium with its electrode, which is equivalent to assume that the adsorbed molecules completely screen the electric field so that the entire potential drop occurs across the vacuum region. This should be an accurate approximation for small applied biases $(V \leqslant 100 \mathrm{mV}$ ) which is the usual picture in IETS experiments. Furthermore, given the large $s$ - $a$ distance, we will assume throughout that the VMs at $s$ and $a$ are decoupled. ${ }^{35}$

Under TRCs the lesser and greater self-energies at $\alpha=s, a$ are simply given by

$$
\Sigma_{\alpha \alpha}^{\gtrless, \gamma}=i n_{\alpha}^{\gtrless} \Gamma_{\alpha \alpha}^{\gamma},
$$


where $n_{\alpha}^{\gtrless}$ is the Fermi-Dirac thermal distribution of occupied and empty electronic states at $\alpha: n_{\alpha}^{<}=f_{\mathrm{FD}}\left(E_{\alpha} / k T\right)$ and $n_{\alpha}^{>}=$ $n_{\alpha}^{<}-1$. In order to account for the applied bias $e V=\mu_{L}-$ $\mu_{R}$, we define the (complex) energies at each molecule as $E_{\alpha}=$ $E-\mu_{\gamma} \pm e V / 2+i \lim _{\delta \rightarrow 0^{+}} \delta$. Following the standard STM convention, we use the + sign for the $e V / 2$ term if $\alpha$ belongs to the sample electrode $(\alpha=s$ and $\gamma=L)$ and the - sign if it belongs to the tip electrode $(\alpha=a$ and $\gamma=R$ ). Although we omit the explicit $E$ dependence throughout most of this section, one must bear in mind that $n_{\alpha}^{\gtrless}$ and the secular matrix terms $F_{\alpha \beta}=E_{\alpha} \delta_{\alpha \beta}-H_{\alpha \beta}$ are the only quantities where the energy $E$ is input.

$\Gamma_{\alpha \alpha}^{\gamma}$ in Eq. (3) is the usual molecule-electrode coupling which, under equilibrium conditions, is given by

$$
\Gamma_{\alpha \alpha}^{\gamma}=i\left[\Sigma_{\alpha \alpha}^{\gamma}-\text { H.c. }\right] \text {. }
$$

The electrode derived retarded self-energies $\Sigma_{\alpha \alpha}^{\gamma}$ can be obtained from

$$
\Sigma_{\alpha \alpha}^{\gamma}=H_{\alpha \gamma} G_{\gamma \gamma}^{s} H_{\gamma \alpha},
$$

where $G_{\gamma \gamma}^{s}$ is the retarded Green's function projection onto the surface of the $\gamma$ electrode without the molecule $\alpha$ and should be calculated taking into account that the electrode is semi-infinite. $^{31}$

We will consider first the case when the leads are decoupled, while in the following sections we will present several approaches to evaluate the $G^{\gtrless \text {,ph }}$ terms.

\section{B. Isolated leads in equilibrium}

If all interlevel interactions are removed $\left(H_{s a}=M_{s a}=0\right)$, the left and right leads decouple and each lead may be treated independently. We will use the $s$ superscript to denote the quantities associated to each isolated lead. The retarded Green's function projection onto molecule $\alpha=a / s, G_{\alpha \alpha}^{s}$, is

$$
G_{\alpha \alpha}^{s}=\left(F_{\alpha \alpha}-\Sigma_{\alpha \alpha}^{\gamma}\right)^{-1} \text {. }
$$

Recalling that each isolated lead is in equilibrium we have

$$
G_{\alpha \alpha}^{\gtrless, s}=i n_{\alpha}^{\gtrless} A_{\alpha \alpha}^{s},
$$

where we have defined the spectral function $A_{\alpha \alpha}^{s}$ by

$$
A_{\alpha \alpha}^{s}=i\left(G_{\alpha \alpha}^{s}-\text { H.c. }\right) \text {. }
$$

It is easy to prove the two following identities, which will become useful later:

$$
\begin{gathered}
i \Gamma_{\alpha \alpha}^{\gamma}=\left[\left(G^{s}\right)^{-1}-\text { c.c. }\right], \\
A_{\alpha \alpha}=G_{\alpha \alpha}^{s} \Gamma_{\alpha \alpha}^{\gamma}\left(G_{\alpha \alpha}^{s}\right)^{\dagger} .
\end{gathered}
$$

We may also include at this point any vibrations at $\alpha$ with the VM dressed Green's function given by

$$
G_{\alpha \alpha}^{s, \mathrm{ph}}=\left(F_{\alpha \alpha}-\Sigma_{\alpha \alpha}^{\gamma}-\Sigma_{\alpha \alpha}^{\mathrm{ph}}\right)^{-1}
$$

with $\Sigma_{\alpha \alpha}^{\mathrm{ph}}$ giving the self-energies arising from the VMs at $\alpha$. Since the molecule is in equilibrium with the lead, Eqs. (7) and (8) can be generalized as

$$
\begin{gathered}
G_{\alpha \alpha}^{\gtrless, s, \mathrm{ph}}=i n_{\alpha}^{\gtrless} A_{\alpha \alpha}^{s, \mathrm{ph}}, \\
A_{\alpha \alpha}^{s, \mathrm{ph}}=i\left(G_{\alpha \alpha}^{s, \mathrm{ph}}-\text { H.c. }\right) .
\end{gathered}
$$

At the SCBA level ${ }^{20}$ and after taking Eq. (12) into account, we arrive at the following set of equations that should be solved self-consistently in conjunction with Eq. (11):

$$
\begin{aligned}
& \Sigma_{\alpha \alpha}^{\gtrless, s, \mathrm{ph}}=i \sum_{\lambda \in \alpha} M_{\alpha \alpha}^{\lambda} \\
& \times\left[N^{\lambda} n_{\alpha}^{\gtrless, \pm \lambda} A_{\alpha \alpha}^{s, p h, \pm \lambda}+N^{\prime \lambda} n_{\alpha}^{\gtrless, \mp \lambda} A_{\alpha \alpha}^{s, p h, \mp \lambda}\right] M_{\alpha \alpha}^{\lambda}, \\
& \Sigma_{\alpha \alpha}^{s, \mathrm{ph}}=\frac{1}{2}\left[\Sigma_{\alpha \alpha}^{>, s, \mathrm{ph}}-\Sigma_{\alpha \alpha}^{<, s, \mathrm{ph}}\right]
\end{aligned}
$$

with $N^{\lambda}$ giving the boson occupation numbers $N^{\lambda}=$ $f_{B E}\left(\Omega_{\lambda} / k T\right)$ and $N^{\prime \lambda}=N^{\lambda}+1$. We have further employed the short notation $n_{\alpha}^{\gtrless, \pm \lambda}=n \gtrless\left(E_{\alpha} \pm \Omega_{\lambda}\right)$ and $A^{s, p h, \pm \lambda}=$ $A^{s, \mathrm{ph}}\left(E \pm \Omega_{\lambda}\right)$. Obviously, for the sample (tip) lead only the VMs at $s(a)$ should be included.

\section{Full order + SCBA approach}

We now consider the general case where the interlevel $H_{s a}$ and $M_{s a}$ interactions have been switched on and a small bias $V$ has been applied. The Green's function of the coupled system projected at the junction may be obtained after inverting the following $(2 \times 2)$ matrix:

$$
\left(\begin{array}{cc}
G_{s s}^{\mathrm{ph}} & G_{s a}^{\mathrm{ph}} \\
G_{a s}^{\mathrm{ph}} & G_{a a}^{\mathrm{ph}}
\end{array}\right)=\left(\begin{array}{cc}
F_{s s}-\Sigma_{s s}^{L}-\Sigma_{s s}^{\mathrm{ph}} & -\Sigma_{s a}-\Sigma_{s a}^{\mathrm{ph}} \\
-\Sigma_{a s}-\Sigma_{a s}^{\mathrm{ph}} & F_{a a}-\Sigma_{a a}^{R}-\Sigma_{a a}^{\mathrm{ph}}
\end{array}\right)^{-1}
$$

with $\Sigma_{s a}=\Sigma_{a s}=H_{s a}=H_{a s}$ and $F_{\alpha \alpha}=E_{\alpha}-H_{\alpha \alpha}$.

The VM related self-energies are coupled to Eq. (16) via the SCBA equations

$$
\begin{aligned}
\Sigma_{\alpha \beta}^{\gtrless, \mathrm{ph}} & =\sum_{\lambda, \mu \nu} M_{\alpha \mu}^{\lambda}\left[N^{\lambda} G_{\mu \nu}^{\gtrless, p h, \pm \lambda}+N^{\prime \lambda} G_{\mu \nu}^{\gtrless, p h, \mp \lambda}\right] M_{\nu \beta}^{\lambda}, \\
\Sigma_{\alpha \beta}^{\mathrm{ph}} & =\frac{1}{2}\left[\Sigma_{\alpha \beta}^{>, \mathrm{ph}}-\Sigma_{\alpha \beta}^{<, \mathrm{ph}}\right]-\frac{i}{2} \mathcal{H}\left\{\Sigma_{\alpha \beta}^{>, \mathrm{ph}}-\Sigma_{\alpha \beta}^{<, \mathrm{ph}}\right\}, \\
G_{\alpha \beta}^{\gtrless, \mathrm{ph}} & =\sum_{\mu \nu} G_{\alpha \mu}^{\mathrm{ph}} \Sigma_{\mu \nu}^{\gtrless}\left(G_{\beta \nu}^{\mathrm{ph}}\right)^{\dagger},
\end{aligned}
$$

where $\mathcal{H}(f)$ stands for the Hilbert transform of $f$ and, in analogy with Eq. (14) we have defined $G_{\alpha \beta}^{\gtrless, p h, \pm \lambda}=$ $G_{\alpha \beta}^{\gtrless, \mathrm{ph}}\left(E_{\alpha \beta} \pm \Omega_{\lambda}\right)$. In the last Eq. (19), $\Sigma_{\mu \nu}^{\gtrless}$ includes all self-energies appearing in the second term of equality (16). The so-called Fock term is not included in the SCBA equations since it is common practice to skip it.

An efficient way to solve self-consistently Eqs. (16)-(19) is to employ as initial conditions the quantities calculated for

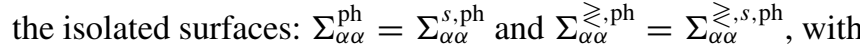
the VM mediated interlevel self-energy matrices initialized to zero: $\Sigma_{\text {sa/as }}^{\mathrm{ph}}=0$.

\section{LOIT + ILSCBA}

A much more efficient approach to solve the 2LS model is to exploit the small values of both $H_{s a / a s}$ and $M_{s a / a s}^{\lambda}$. To this end, we first expand the Dyson equation for the interface 
Green's function up to first order in $\Sigma_{\text {sa/as }}$ :

$$
\begin{gathered}
G_{\alpha \alpha}^{\mathrm{ph}} \approx G_{\alpha \alpha}^{s, \mathrm{ph}}, \\
G_{\alpha \beta}^{\mathrm{ph}} \approx G_{\alpha \alpha}^{s, \mathrm{ph}} \Sigma_{\alpha \beta} G_{\beta \beta}^{s, \mathrm{ph}} \text { for } \alpha \neq \beta .
\end{gathered}
$$

We next apply the first-order Born approximation (BA1) to the inelastic tunneling self-energies $\Sigma_{s a / s a}^{\mathrm{ph}}$ instead of its selfconsistent version (17)-(19). This approach is justified since, in general, not only $H_{s a}$, but also $M_{s a}^{\lambda}$ are small with respect to the intralevel interactions $H_{s s}$ and $H_{a a}$. Still, Eq. (17) leads to a rather large number of terms to be summed over. Fortunately, after inspecting their values, one finds that by far the dominant contributions introduced by the inelastic tunneling are those quadratic in $M_{s a}^{\lambda}$. These terms, denoted hereafter by $\Sigma^{\gtrless, i t}$, take the form

$$
\begin{aligned}
\sum_{\alpha \alpha}^{\gtrless, i t}= & i \sum_{\lambda} M_{\alpha \beta}^{\lambda}\left[N^{\lambda} n_{\beta}^{\gtrless, \pm \lambda} A_{\beta \beta}^{s, p h, \pm \lambda}\right. \\
& \left.+N^{\prime \lambda} n_{\beta}^{\gtrless, \mp \lambda} A_{\beta \beta}^{s, p h, \mp \lambda}\right] M_{\beta \alpha}^{\lambda} .
\end{aligned}
$$

The self-energies of the coupled system may then be approximated by

$$
\begin{aligned}
& \Sigma_{\alpha \alpha}^{\gtrless, p h} \approx \Sigma_{\alpha \alpha}^{\gtrless, s, p h}+\Sigma_{\alpha \alpha}^{\gtrless, i t}, \\
& \Sigma_{\alpha \beta}^{\gtrless, p h} \approx 0 \text { for } \alpha \neq \beta .
\end{aligned}
$$

Under approximations (20) through (24), the lesser and greater Green's functions may be expressed as

$$
\begin{aligned}
G_{\alpha \alpha}^{\gtrless, \mathrm{ph}}= & G_{\alpha \alpha}^{\gtrless, s, \mathrm{ph}}+G_{\alpha \alpha}^{s, \mathrm{ph}} \Sigma_{\alpha \alpha}^{\gtrless, i t}\left(G_{\alpha \alpha}^{s, \mathrm{ph}}\right)^{\dagger} \\
& +G_{\alpha \beta}^{\mathrm{ph}} \Sigma_{\beta \beta}^{\gtrless, s, \mathrm{ph}}\left(G_{\alpha \beta}^{\mathrm{ph}}\right)^{\dagger} \quad \text { with } \quad \alpha \neq \beta .
\end{aligned}
$$

Equations (22)-(25) constitute our lowest order in inelastic tunneling (LOIT) approximation. Notice that all terms involved include just one tunneling event, either elastic or inelastic. Its accuracy and limits of applicability will be discussed below in Sec. II G.

With the use of Eqs. (3) and (25) and after some algebra, the differential current in (2) may be written as

$$
g_{\alpha}(E, V)=\operatorname{Tr}\left[\left(\hat{\rho}_{\alpha \alpha}+\hat{\rho}_{\alpha \alpha}^{i t}\right) \Gamma_{\alpha \alpha}^{\gamma, \mathrm{ph}}\right]
$$

with

$$
\begin{gathered}
\hat{\rho}_{\alpha \alpha}=\left(n_{\alpha}^{<}-n_{\beta}^{<}\right) G_{\alpha \beta}^{\mathrm{ph}}\left(\Gamma_{\beta \beta}^{\gamma}+\Gamma_{\beta \beta}^{s, \mathrm{ph}}\right)\left(G_{\alpha \beta}^{\mathrm{ph}}\right)^{\dagger}, \\
\hat{\rho}_{\alpha \alpha}^{i t}=i G_{\alpha \alpha}^{s, \mathrm{ph}}\left(n_{\alpha}^{<} \Sigma_{\alpha \alpha}^{>, i t}-n_{\alpha}^{>} \Sigma_{\alpha \alpha}^{<, i t}\right)\left(G_{\alpha \alpha}^{s, \mathrm{ph}}\right)^{\dagger} .
\end{gathered}
$$

Although the $p h$ superscript should not appear in the electrode derived broadening $\Gamma_{\alpha \alpha}^{\gamma, \text { ph }}$ in Eq. (26), its insertion yields a current-conserving formula and in selected cases it also improves the accuracy of the approximation.

The interpretation of the above formula is straightforward if we identify $\Gamma_{\alpha \alpha}^{\gamma}$ as the velocity operator towards the $\gamma$ electrode and $\hat{\rho}\left(\hat{\rho}^{i t}\right)$ as the net local density of propagating states towards the electrode assuming only elastic (inelastic) tunneling. Since intralevel (IL) inelastic transitions are treated at the SCBA level, we will denote Eqs. (27) and (28) as the LOIT + ILSCBA approach.

\section{E. LOIT}

Since in general the intralevel $e$-VM terms $M_{\alpha \alpha}^{\lambda}$ will be larger than the inelastic tunneling terms $M_{\alpha \beta}^{\lambda}$, one would naively expect that the influence of the former in any IETS curve is substantially larger than the latter. However, this is by no means the case and from the test calculations (to be shown in the following), we find that, in fact, the intralevel $e$-VM couplings can be safely removed in most cases. Under this approximation, the surface Green's functions projected on the levels $G_{\alpha \alpha}^{s}$ need not be dressed by the VMs any more and one can write the differential current fully decoupling the purely elastic term $g_{\alpha}^{e l}$ from that arising solely from the inelastic tunneling $g_{\alpha}^{i t}$ :

$$
g_{\alpha}=g_{\alpha}^{e l}+g_{\alpha}^{i t} .
$$

Inserting the first-order approximation (21) and the LOIT Eqs. (22) and (25) into (27) and (28) after dropping the ph superscript and making use of identities (9) and (10), we finally arrive at the compact expressions

$$
\begin{aligned}
g_{\alpha}^{e l}= & \frac{2 e}{h}\left(n_{\alpha}^{<}-n_{\beta}^{<}\right) \operatorname{Tr}\left[A_{\alpha \alpha}^{s} F_{\alpha \beta} A_{\beta \beta}^{s}\left(F_{\alpha \beta}\right)^{\dagger}\right] \\
g_{\alpha}^{i t}= & -\frac{2 e}{h} \times\left[A _ { \alpha \alpha } ^ { s } \left(\sum _ { \lambda } M _ { \alpha \beta } ^ { \lambda } \left[\left(f_{\alpha \beta}^{e,-\lambda}-f_{\alpha \beta}^{a,-\lambda}\right) A_{\beta \beta}^{s,-\lambda}\right.\right.\right. \\
& \left.\left.\left.+\left(f_{\alpha \beta}^{a,+\lambda}-f_{\alpha \beta}^{e,+\lambda}\right) A_{\beta \beta}^{s,+\lambda}\right] M_{\beta \alpha}^{\lambda}\right)\right]
\end{aligned}
$$

Expression (31) shows that the inelastic tunneling signal is quadratic in the $e$-VM couplings $M_{\alpha \beta}^{\lambda}$ and allows us to decompose the inelastic signal in terms of the contribution of each individual VM. The bias-dependent emission and absorption factors $f_{\alpha \beta}^{e / a, \pm \lambda}$ are defined as

$$
\begin{aligned}
& f_{\alpha \beta}^{a, \pm \lambda}=n_{\alpha}^{\lessgtr} N^{\lambda} n_{\beta}^{\gtrless, \pm \lambda}, \\
& f_{\alpha \beta}^{e, \pm \lambda}=n_{\alpha}^{\gtrless} N^{\prime \lambda} n_{\beta}^{\lessgtr, \pm \lambda} .
\end{aligned}
$$

Their physical meaning is sketched in Fig. 2. The absorption terms $f_{\alpha \beta}^{a, \pm \lambda}$ are linear in $N^{\lambda}$ and since at the usual temperatures employed in IETS experiments $(k T<10 \mathrm{meV})$ the VMs are essentially depopulated, their contribution to $g_{\alpha}^{i t}$ is in general negligible: only those VMs with very low frequencies (below $k T$ ) contribute to the inelastic features. The emission factors $f_{\alpha \beta}^{e, \pm \lambda}$ are, on the other hand, the dominant terms as they open inelastic channels once $|V|>\Omega_{\lambda}$, thus leading to peak/dip features at positive/negative values (see following).

\section{F. $\mathrm{LOIT}+\mathrm{WBL}$}

We may further assume that the electronic structure of each lead behaves smoothly around the Fermi level. One may then adopt the so-called wide band limit (WBL) whereby all
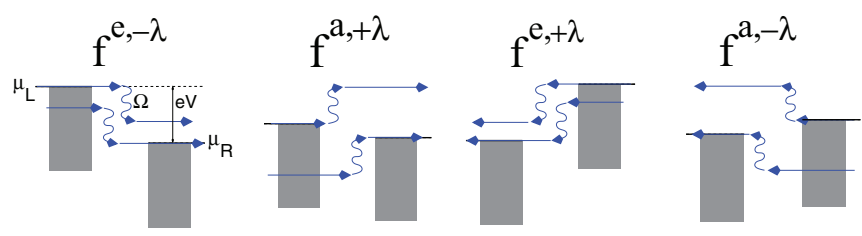

FIG. 2. (Color online) Sketch of the four types of inelastic tunneling processes included in the LOIT approximation, corresponding to the factors in (32) and (33). 
Green's function related terms are assumed constant within the bias window and may be evaluated only at the Fermi level $\mu_{L / R}$. Under the WBL, the only terms that depend on energy will be those related to the fermion occupations so that we may reexpress the inelastic contribution to the current $I^{i t}(V)$ in a computationally optimal way:

$$
\begin{aligned}
I^{i t}(V) & =\frac{2 e}{h} \operatorname{Tr}\left[A_{\alpha \alpha}^{s}\left(\sum_{\lambda} \Gamma_{\alpha \alpha}^{i t, \lambda} f_{\alpha \beta}^{i t, \lambda}\right)\right], \\
\Gamma_{\alpha \alpha}^{i t, \lambda} & =M_{\alpha \beta}^{\lambda} A_{\beta \beta}^{s} M_{\beta \alpha}^{\lambda}, \\
f_{\alpha \beta}^{i t, \lambda} & =\int d E\left(f_{\alpha \beta}^{e,+\lambda}-f_{\alpha \beta}^{a,+\lambda}+f_{\alpha \beta}^{a,-\lambda}-f_{\alpha \beta}^{e,-\lambda}\right) .
\end{aligned}
$$

Recall that the energy $E$ only enters the integral in Eq. (36), while the rest of terms are evaluated at the Fermi level. Other related approximations are to freeze the electronic structure only at one of the leads, either the tip or the sample.

\section{G. LOIT versus SCBA}

Once we have formulated the LOIT approximation, we now study its accuracy by applying it to the 2LS model of Fig. 1(b) and comparing the results against those obtained under the more accurate SCBA approach. We will constrain the parameter space to be explored to values that mimic a generic STM setup with metallic electrodes. First, we assume that the WBL holds for both $L$ and $R$, so that Eqs. (4) and (5) reduce to $\Gamma_{\alpha \alpha}^{\gamma}=4 H_{\alpha \gamma} / \Gamma_{\gamma}$, where $\Gamma_{\gamma}$ is the broadening at the electrode surface, which should be of the order of the eV. In practice, we fix $\Gamma_{L}=1 \mathrm{eV}$, while $H_{s L}$ is either increased or decreased in order to model a chemisorbed or physisorbed level at the surface. The molecule onsite energy is fixed as well to $H_{s s}=80 \mathrm{meV}$ in order to address both the on- and off-resonance regimes (see below). For the tip, we will assume that the $a$ level is chemisorbed $\left(H_{a R}=4 \mathrm{eV}\right)$ with onsite energy $H_{a a}=-80 \mathrm{meV}$, tuned to open a resonant tunneling channel with the $s$ level at a bias of $V=-80 \mathrm{meV}$. A low VM frequency of $\Omega_{s}=30 \mathrm{meV}$ is also assumed at $s$ throughout. In all simulations, TRCs are preserved by only considering tip-sample interactions satisfying $H_{s a} / \Gamma_{\alpha \alpha}^{\gamma} \leqslant 0.1$ and $M_{s a}^{\lambda} / M_{s s}^{\lambda} \leqslant 0.1$ for the $e$-VM couplings.

Simulated $\left(\partial^{2} I / \partial V^{2}\right) /(\partial I / \partial V)$ IETS curves for four different sets of parameters are presented in Fig. 3. In Fig. 3(a), a typical off-resonance regime is considered after assuming that $s$ is chemisorbed $\left(H_{s L}=4 \mathrm{eV}\right)$. Despite the rather large value of the intralevel $e$-VM coupling $\left(M_{s s}^{\lambda}=100 \mathrm{meV}\right)$, the LOIT approximation (blue line) is essentially exact compared to the SCBA calculation (dark line), showing a sharp peak (dip) at positive (negative) biases $(V= \pm 30 \mathrm{mV})$. The regime plotted in Fig. 3(b) is somewhat a generalization of the previous case after adding a VM at the tip $\left(\Omega_{a}=10 \mathrm{meV}\right)$. The LOIT approach is still very close to the SCBA, both showing larger peak/dip heights for the $s$ level than for the $a$ level since we have set smaller $e$-VM couplings at $a$ than at $s$. The subtle differences between both approaches are due to an asymmetric contribution in the SCBA curve which now becomes visible due to the decreased value of $H_{S L}=2 \mathrm{eV}$. Since the asymmetry arises from the Hilbert transform ${ }^{20}$ in Eq. (18), neither the LOIT nor the LOIT + ILSCBA, which do not include this transform, can reproduce it. Most interestingly, if the interlevel
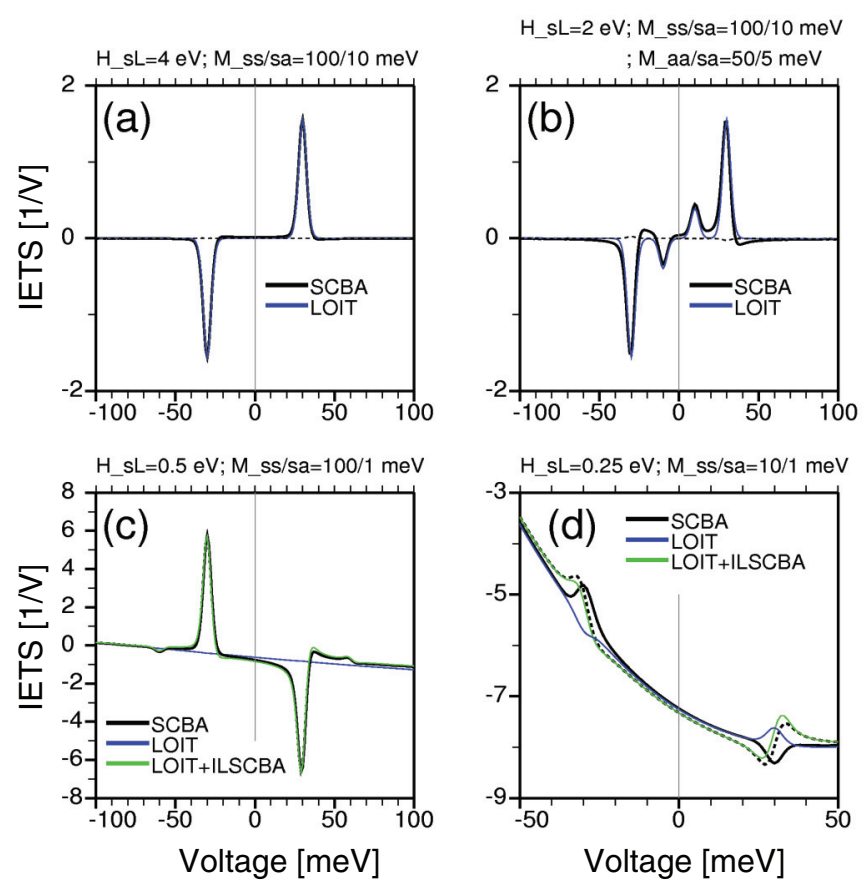

FIG. 3. (Color online) IETS spectra for the 2LS model depicted in Fig. 1 obtained with different sets of parameters, as indicated at the top of each graph. (a) and (b) Off-resonance regime including one and two VMs, respectively. (c) and (d) On-resonance regimes after reducing $H_{s L}$. Black, blue, and green lines are used for the SCBA, LOIT, and LOIT + SCBA approximations, respectively. See text for further explanations. Dashed lines correspond to SCBA calculations after setting $M_{s a}=0$.

$e$-VM coupling is switched off by setting $M_{a s}^{\lambda}=0$ [dashed line in Figs. 3(a) and 3(b)], the inelastic traces are not apparent any more, proving that for this regime the IETS features are completely ruled by the inelastic tunneling processes and, hence, the high accuracy of the LOIT approximation. This is further corroborated by noting that the curves for the LOIT + ILSCBA approach (not shown) are essentially identical to the LOIT ones.

Figure 3(c) shows an on-resonance case, with a sharp dip (peak) at positive (negative) biases. To this end, we have assumed a weakly adsorbed molecule $H_{s L}=500 \mathrm{meV}$, and decreased substantially the tunneling $e$-VM coupling to $M_{s a}=1 \mathrm{meV}$. This time the LOIT fails to give any inelastic features since, given the small $M_{s a}^{\lambda}$ value, the intralevel scattering processes at $s$ become dominant. However, the ILSCBA correction (green curve) provides an excellent agreement with the full SCBA curve, even reproducing the small satellite peaks at $V= \pm 60 \mathrm{mV}$ arising from two-phonon processes. In Fig. 3(d) the spectra have been calculated for the same parameters except after further reducing the electrode-molecule interaction (physisorption limit) as well as the intralevel $e$-VM coupling to $M_{s s}^{\lambda}=10 \mathrm{meV}$. The former increases the intralevel inelastic contribution, but the latter has the opposite effect and is equivalent to increasing the strength of the inelastic tunneling processes. The curves show a large curvature due to the elastic resonant peak at $V=$ $-80 \mathrm{mV}$ (not shown), while the inelastic features are of smaller magnitude. The dip/peak structure at positive/negative biases 
is at contrast with the LOIT approach and is not even accurately predicted by the LOIT + ILSCBA, which yields a rather symmetric step at both polarities. The reason is the relative enhancement of the inelastic tunneling contribution, which now competes and entangles with the intralevel scattering and invalidates the LOIT approximation given by Eqs. (22)-(25). Indeed, if the inelastic tunneling channel is suppressed in the SCBA calculation (dashed line), we retrieve the situation of Fig. 3(c) and the ILSCBA correction becomes again very accurate.

After extensive tests, we have found the general trend that the LOIT approach is highly precise whenever the levels interact strongly with the electrodes (large $H_{S L / a R}$ values); in this case the inelastic tunneling contribution, being quadratic in $M_{s a}^{\lambda}$, largely dominates the IETS spectra even for large intralevel $e$-VM couplings [off-resonance case in Figs. 3(a) and $3(\mathrm{~b})$ ]. As we reduce the level-electrode interaction, the intralevel inelastic contribution is enhanced and the leading inelastic events now include cross terms $M_{s s}^{\lambda} \times M_{s a}^{\lambda}$ [Fig. 3(d)]. In this case, neither the LOIT nor the ILSCBA corrections are accurate and the full SCBA approach (or its first-order version) is required. In the limit where $M_{s a}^{\lambda} \ll M_{s s}^{\lambda}$ and the level interacts weakly with the electrode, the LOIT + ILSCBA approach becomes accurate [on-resonance case in Fig. 3(c)]. Anyhow, we should recall that in the on-resonance regime (i) the levels need not be in equilibrium with their respective electrodes anymore, (ii) the Fock term, which has been neglected in the evaluation of the VM related self-energies $\Sigma^{\mathrm{ph}}$ may induce significant polaronic shifts to the IETS features, and (iii) small $H_{S L}$ values in the STM model may leave the $s$ level in a Coulomb blockade regime for which the transport formalism applied here is not valid.

In summary, we find that the LOIT is an excellent approximation to the simulation of STM-IETS spectra as long as the level (molecule) is chemisorbed at the surface and/or apex. Although this fact seems counterintuitive (in general the inelastic intralevel scattering will be larger than the tunneling one $M_{s a}^{\lambda} \ll M_{s s}^{\lambda}$ ), it may be rationalized by recalling the elastic analog where the tip-sample interaction is the most relevant term in determining the topography in an STM image, despite it is typically much smaller than the electrode derived self-energies $\left(H_{s a} \ll \Gamma_{s s}^{\gamma}\right)$. On the other hand, when the molecule is weakly coupled to the electrode, the LOIT is not precise any more and an accurate calculation of the elastic and inelastic transport properties becomes highly challenging and beyond the scope of this work.

\section{3D STM MODEL}

In this section, we generalize the LOIT formalism to a realistic three-dimensional (3D) model for the STM system. We adopt the rather general geometrical picture employed within the GREEN (Refs. 31 and 32) code which has been extensively used for STM topographic simulations. A side view of the STM interface model is depicted in Fig. 4(a). The sample and tip electrodes are modeled by semi-infinite twodimensional (2D) bulk blocks. Each bulk block is itself split into a semi-infinite stack of principal layers (PLs); typically, a PL comprises several atomic planes. Molecules $s$ and $a$ in the 2LS are now described by the sample surface and tip-apex
PLs, respectively. $s$ includes one or two reconstructed surface layers plus any adsorbates and is assumed 2D periodic with a unit cell which should be commensurate to that of the sample electrode. On the other hand, the tip apex $a$ is considered nonperiodic (isolated in 2D) and consists of a pyramid of 9 to 11 atoms stacked below the tip bulk.

To describe the electronic part of the system, we employ a linear combination of atomic orbitals (LCAO) basis set as implemented in the SIESTA (Ref. 33) code. The generalization of the LOIT derived Eqs. (30) and (31) to the 3D case is straightforward after taking into account the following:

(i) All Green's functions related projections onto any pair of PLs $(\alpha, \beta)$ will be matrices in the $\mathrm{AO}$ basis set.

(ii) The secular matrix of the entire chain $F$ now includes the overlap matrix $S: F_{\alpha \beta}(E)=E_{\alpha \beta} S_{\alpha \beta}-H_{\alpha \beta}$ where the block-dependent (complex) energies $E_{\alpha \beta}$ are

$$
E_{\alpha \beta}=E-\frac{1}{2}\left(\mu_{\gamma}+\mu_{\gamma^{\prime}} \pm e V \delta_{\gamma \gamma^{\prime}}\right)+i \lim _{\delta \rightarrow 0^{+}} \delta,
$$

with $\gamma\left(\gamma^{\prime}\right)$ being the electrode to which $\alpha(\beta)$ is attached.

(iii) Since the two electrodes as well as the sample surface $s$ are assumed periodic in two dimensions, we construct $\vec{k}$-dependent matrices in these PLs in order to sample the associated Brillouin zones. The apex PL, on the other hand, is represented by a single $\Gamma$ point which couples all $\vec{k}$ points used to sample the tip electrode.

Next, we will describe how the evaluation of the matrices necessary to obtain both the elastic and inelastic current across the STM junction have been implemented in the upgraded version of the GREEN code. A flow chart indicating the sequence of the steps to be carried out for an IETS simulation under our $a b$ initio LOIT scheme is given in Fig. 4(g).

\section{A. Hamiltonian}

Clearly, the system depicted in Fig. 4(a) is far too complex to be calculated within a unique self-consistent $a b$ initio calculation. Instead, we perform different SIESTA calculations for different parts of the system as sketched in the figure and store their Hamiltonian matrix elements so that they can be used at any point in the Green's functions calculations. ${ }^{36,37}$ We assume that all inter-PL interactions only extend up to first-nearest neighbors. Given the strict localization of the AOs, this is always achievable by including enough atomic layers in the PLs to ensure that all interactions among nonadjacent PLs are zero (typical PL thicknesses are around 6-10 $\AA$ ). Figure 4 shows the structure of the resulting tridiagonal (infinite) Hamiltonian matrix, where each box corresponds to the interaction between two PLs. The boxes are grouped into five colored cages, each associated to a different SIESTA calculation:

(i) Bulk calculations for both the tip and the sample [Figs. 4(b) and 4(c), respectively] giving all interactions within the electrodes: $H_{\gamma \gamma}$ and $H_{\gamma \gamma}^{ \pm}$matrix blocks. These two calculations are quite simple since they typically involve one or just a few atoms in the bulk unit cell.

(ii) Sample surface calculation [Fig. 4(e)] for the $H_{s s}$ and $H_{s L / L s}$ matrix blocks. Here, we consider a slab including the $s$ PL, and we add several bulk layers below in order to ensure that a bulk environment is achieved at the center of the slab. ${ }^{37}$ The 

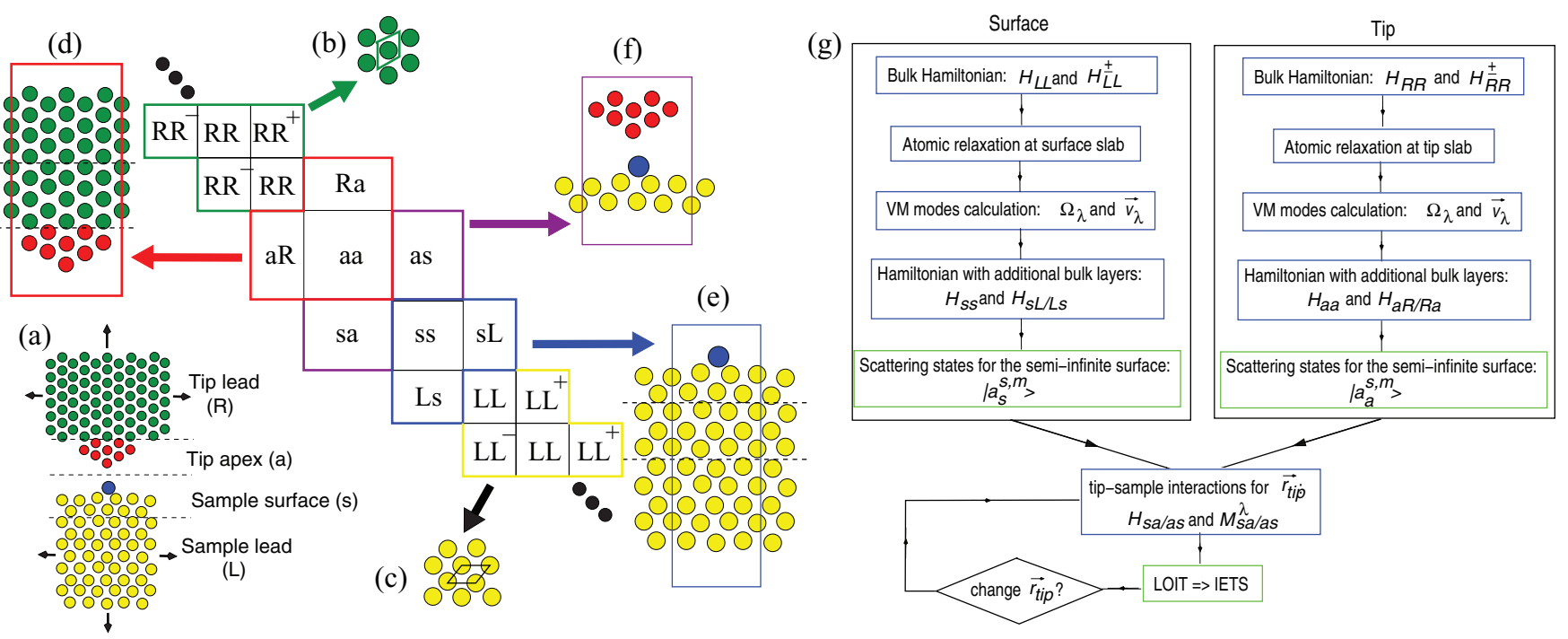

FIG. 4. (Color online) (a) Side view for the complete 3D STM model. Arrows indicate that the system extends to infinity along that direction. (b)-(f) Sketch of the infinite tridiagonal Hamiltonian matrix describing the entire STM system together with side views of the different systems (calculated at the $a b$ initio level) required to obtain all matrix elements. Each box corresponds to a matrix block $H_{\alpha \beta}$ holding the interactions between PLs $\alpha$ and $\beta$ in the AO basis set. Thick colored bounding boxes and arrows are used to indicate from which ab initio calculation are the matrix elements extracted: (b) tip bulk, (c) sample bulk, (d) tip apex, (e) sample surface, and (f) tip surface. (g) Flow chart for our LOIT ab initio scheme. Blue (green) boxes refer to calculations performed under SIESTA (GREEN). See text for further explanations.

extra layers are essential to ensure that the entire formalism is self-consistent.

(iii) Tip-apex calculation [Fig. 4(d)] for the $H_{a a}$ and $H_{a R / R a}$ matrix blocks. Same as in (ii), we add on top of $a$ further tip bulk layers. Although the calculations are typically performed for a $(4 \times 4)$ or $(3 \times 3)$ supercell, we drop all matrix elements related to apex atoms outside the origin cell when loading the $H_{a a}$ and $H_{a R / R a}$ matrices in the Green's function calculation since the apex is assumed nonperiodic.

(iv) The apex-surface interactions $H_{s a / a s}$ are obtained from the slab geometry given in Fig. 4(f). Apart from the adsorbates we include a few surface layers (two or three) and stack on top the apex atoms at the desired relative position.

\section{B. Equilibrium geometry and vibrational modes}

A main advantage of the LOIT approach is that the VMs at the surface and the tip are fully decoupled. This allows their evaluation at the $a b$ initio level via independent SIESTA calculations, thus reducing considerably the computational cost. One first needs to obtain the equilibrium geometries to be used in the slab calculations (ii) and (iii). In general, this optimization is performed for the same slabs as those depicted in Figs. 4(d) and 4(e) but reducing the number of bulk layers to four of five. During the relaxations, only the atoms in PLs $s$ or $a$ are allowed to relax while the rest are fixed to bulk positions. The resulting optimized coordinates are then used to construct the large slab for which only one (computationally heavy) self-consistent calculation is required at the end of which the Hamiltonian is saved to disk.

The vibrational modes (VMs) are obtained after constructing the mass-scaled dynamical matrix $C_{I \mu J v}$ according to

$$
C_{I \mu J v}=\frac{1}{\sqrt{M_{I} M_{J}}} \frac{\partial F_{I \mu}}{\partial R_{J v}},
$$

where $I / J$ denote the dynamic atoms with (equilibrium) position vectors $\vec{R}_{I / J}$ and atomic masses $M_{I / J} . \mu / v$ refer to the Cartesian coordinates and $F_{I \mu}$ is the force on atom $I$ along $\mu$. The vibrational frequencies $\Omega_{\lambda}$ and eigenmodes $\vec{v}^{\lambda}$ are then obtained after diagonalization of the dynamical matrix..$^{20}$ As usual, any negative eigenvalues (imaginary frequencies) arising from accuracy errors are discarded.

Since the forces are readily obtained from a standard SIESTA calculation, we approximate the derivatives appearing in Eq. (38) numerically by displacing the dynamic atoms within $a$ or $r$ from their equilibrium positions by a finite amount $\delta r$ along the three Cartesian coordinates $\mu=x, y, z$. Obviously, we employ the same cell as that used for the geometry optimization, while the diagonal elements $C_{I \mu I \mu}$ are corrected following the prescription of Ref. 20. When the size of the unit cell is large, the calculation of the partial derivatives may become rather time consuming due to the elevated number of degrees of freedom. In such cases, we freeze those substrate atoms within PL $s$ which are far away (further than $\sim 5 \AA$ ) from the adsorbed molecule. On the other hand, VMs at the tip are only calculated if a molecule/atom is adsorbed at the apex (contaminated tips).

\section{Tip-sample interactions and $e$-VM coupling}

We describe next how the tip-surface matrices $H_{s a / a s}$ and $M_{\text {sa/as }}^{\lambda}$ may be calculated in a single run. To this end, we construct the 2D periodic slab depicted in Fig. 4(f) comprising a few surface layers and placing the apex atoms above them at any desired location $\vec{r}_{\text {tip }}$. The 2D unit cell typically corresponds to the surface supercell unless it is too small to accommodate all the apex atoms. In such cases, we need to define a new supercell commensurate to the surface one comprising replicas of the former and which should be chosen large enough to 
ensure that the apex atoms do not overlap with those in the neighboring cells. The slab normal vector may be defined either choosing an appropriate length so that the top of the apex binds somehow to the bottom of the surface or, alternatively, leaving a vacuum region between both blocks: the latter case is the one depicted in Fig. 4(f). We have found that the results are hardly modified by this particular choice. After a selfconsistent calculation for this cell, the matrices $H / M_{s a / a s}^{\lambda}$ are stored in disk so that they can be used in the calculation of the current as described in the next section.

The same slab is employed for the evaluation of the tipsurface $e$-VM coupling matrices. The matrix elements $M_{i j}^{\lambda}$ for $\mathrm{VM} \lambda$ and between AOs $i$ and $j$ are given by

$$
M_{i j}^{\lambda}=\sum_{I \nu} H_{i j}^{\prime I v} v_{I \nu}^{\lambda} \sqrt{\frac{\hbar}{2 M_{I} \Omega_{\lambda}}},
$$

where $v_{I v}^{\lambda}$ is the $\lambda$ eigenmode component for the $v$ coordinate of atom $I$ and we have defined $H_{i j}^{\prime} I v=\left\langle i\left|\frac{\partial H}{\partial R_{I V}}\right| j\right\rangle$ as the partial derivatives of the Hamiltonian operator with respect to each degree of freedom. Obviously, the summation over $I$ only includes the dynamic atoms.

To evaluate the $H_{i j}^{\prime} I v$ terms, we first note that the SIESTA Hamiltonian may be written as

$$
H=T+V^{\mathrm{KB}}+H^{\mathrm{SCF}},
$$

where $T$ corresponds to the kinetic operator, $V^{\mathrm{KB}}$ to the pseudopotentials in their Kleinman-Bylander form, ${ }^{38}$ while $H^{\mathrm{SCF}}$ includes the Hartree and exchange-correlation potentials and is the only term that depends on the electron density and thus needs to be evaluated self-consistently. It is trivial to show that neither $T$ nor $V^{\mathrm{KB}}$ give any contribution to the Hamiltonians partial derivatives, so that $H_{i j}^{\prime I \nu}=\left\langle i\left|\frac{\partial H^{\mathrm{SCF}}}{\partial R_{I v}}\right| j\right\rangle$. This form allows a fast numerical evaluation of the $e$-VM coupling once the potential $V^{\mathrm{SCF}}(\vec{r})$ for the equilibrium geometry has been solved self-consistently on a real-space grid. For each degree of freedom $I v$, we displace atom $I$ from its equilibrium position by a small amount $\left(\delta I v= \pm 10^{-3} \AA\right)$ along the $\pm v$ coordinate, update the charge density $\rho(\vec{r})$, and calculate the shifted potentials $V_{ \pm \delta I v}^{\mathrm{SCF}}(\vec{r})$. In the calculation of $V_{ \pm \delta I v}^{\mathrm{SCF}}$, and given the small displacements, all the timeconsuming grid-related sparse information remains unchanged and only the wave-function values of atom $I$ on the grid require reevaluation. On the other hand, no self-consistency is required since the density matrix is assumed unchanged after the small atomic displacements. The $H_{i j}^{\prime}{ }^{I v}$ matrix elements are finally obtained via numerical derivatives after transforming the shifted potentials into the AO basis representation. We have checked that this procedure yields very similar results to those obtained with the approach followed in Ref. 20 despite ours is orders of magnitude more efficient.

The scheme provides both the intralead $M_{\alpha \alpha}^{\lambda}$ and interlead $M_{\alpha \beta}^{\lambda}$ matrices in the same run. Although we will discard the former throughout, we recall that for an LOIT + ILSCBA calculation it would be more efficient and accurate to obtain the $M_{\alpha \alpha}^{\lambda}$ terms performing similar independent calculations for the surface and/or the tip slabs described in (ii) and (iii) instead.
Let us finally note that the current step is the least accurate one in the entire ab initio scheme since the $H / M_{\text {sa }}^{\lambda}$ as matrix elements are calculated under 2D periodic boundary conditions, while for the actual STM calculation, as the apex is assumed nonperiodic, all interactions involving apex atoms in neighbor cells are discarded. Our approach also partly neglects the self-energies at $s$ and $a$ arising from the electrodes. Achieving a strict self-consistency for these matrix elements will require an even larger 2D supercell plus the addition of further bulk atoms at the top and bottom of the slab. Still, we believe the slabs employed in this work remain within reasonable limits of accuracy and computational cost given the complexity of the STM interface.

\section{Current}

For computational efficiency, it is highly advantageous to decompose the spectral matrices given in Eq. (8) into diagonal form:

$$
A_{\alpha \alpha}^{s}=\sum_{m_{\alpha}}\left|a_{\alpha}^{s, m_{\alpha}}\right\rangle\left\langle a_{\alpha}^{s, m_{\alpha}}\right|,
$$

where the kets $\left|a_{\alpha}^{s, m_{\alpha}}\right\rangle$ are the eigenvectors normalized by the square root of the associated eigenvalue and may be identified with the scattering states within each lead. The spectral decomposition reduces significantly the computational load since only those scattering states with a non-negligible eigenvalue need to be retained.

Substituting Eq. (41) into Eqs. (30) and (31), we arrive at a Fermi's golden rule type expressions for the differential currents:

$$
\begin{aligned}
g_{\alpha}^{e l}= & \left(n_{\alpha}^{<}-n_{\beta}^{<}\right) \sum_{m_{\alpha}, m_{\beta}}\left|\left\langle a_{\alpha}^{s, m_{\alpha}}\left|F_{\alpha \beta}\right| a_{\beta}^{s, m_{\beta}}\right\rangle\right|^{2} ; \quad \alpha \neq \beta \\
g_{\alpha}^{i t}= & \sum_{m_{\alpha}, m_{\beta}, \lambda}\left[\left(f_{\alpha \beta}^{a,-\lambda}-f_{\alpha \beta}^{e,-\lambda}\right)\left|\left\langle a_{\alpha}^{s, m_{\alpha}}\left|M_{\alpha \beta}^{\lambda}\right| a_{\beta}^{s, m_{\beta},-\lambda}\right\rangle\right|^{2}\right. \\
& \left.+\left(f_{\alpha \beta}^{e,+\lambda}-f_{\alpha \beta}^{a,+\lambda}\right)\left|\left\langle a_{\alpha}^{s, m_{\alpha}}\left|M_{\alpha \beta}^{\lambda}\right| a_{\beta}^{s, m_{\beta},+\lambda}\right\rangle\right|^{2}\right] ; \quad \alpha \neq \beta .
\end{aligned}
$$

Notice that Eq. (42) corresponds to a first-order version of the Landauer-Büttiker ${ }^{39}$ formula. The appealing part of assuming TRCs is that in Eqs. (42) and (43) the scattering states $\left|a^{s, m_{\alpha}}\right\rangle$ are intrinsic to each lead and do not depend on the relative tip-sample position $\vec{r}_{\text {tip. }}$. Therefore, they can be calculated independently for the sample surface and each of the tips to be considered and stored in disk as a function of energy and $k$ points. ${ }^{32}$ During the actual STS/IETS simulation, these vectors will be read in together with the $H_{\alpha \beta}$ and $M_{\alpha \beta}^{\lambda}$ matrices corresponding to the desired tip-sample relative displacement.

\section{CALCULATION PARAMETERS}

Although the LOIT formalism presented in the previous section is $a b$ initio at the DFT level, there are still several parameters entering the calculation the effects of which need to be addressed. We may distinguish those parameters specific to the SIESTA calculations from those entering the Green's functions. In order to test their performance, we have chosen a widely studied system such as $\mathrm{CO}$ adsorption on 

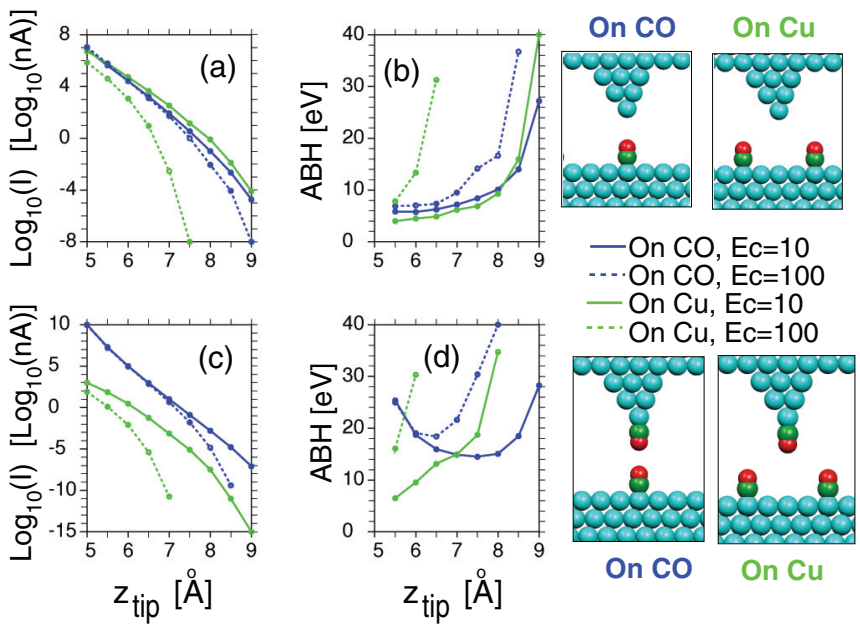

FIG. 5. (Color online) (a) $\log _{10} I\left(z_{\text {tip }}\right)$ curves simulated for a sharp $\mathrm{Cu}(111)$ tip probing a $\mathrm{CO}$ molecule on a $\mathrm{Cu}(111)$ surface (see sketch at the right). Solid (dashed) lines correspond to calculations with extended (contracted) AO basis for which $E_{c}$ was set to $10 \mathrm{meV}$ $(100 \mathrm{meV})$. Blue (green) lines correspond to the apex located on top of the $\mathrm{CO}(\mathrm{Cu}$ substrate). (b) The $\mathrm{ABH}$ values corresponding to the curves shown in (a). (c), (d) Same as (a) and (b) but for a CO contaminated tip, as shown in the sketch at the right. In all cases, the origin in $z_{\text {tip }}$ has been placed at the $\mathrm{Cu}(111)$ surfacemost layer and the bias has been fixed to $70 \mathrm{mV}$.

the $\mathrm{Cu}(111)$ surface. ${ }^{13,14,34,35,40}$ The $\mathrm{CO}$ is known to adsorb at a top site, ${ }^{35}$ despite that DFT does not always identify this site as the most stable one, with the $\mathrm{C}$ attached to the metal atom. Here, we model the surface via a $(4 \times 4)$ supercell. The VM spectra for the adsorbed system consists of a high-frequency molecular stretch mode $\left(v_{\mathrm{CO}} \sim 250 \mathrm{meV}\right)$, another stretch mode between the molecule and the metal $\left(v_{\mathrm{CO}-\mathrm{Cu}} \sim 50 \mathrm{meV}\right)$, frustrated rotations $\left(\mathrm{FR}_{\mathrm{CO}} \sim 30 \mathrm{meV}\right)$, and frustrated translations $\left(\mathrm{FT}_{\mathrm{CO}}<10 \mathrm{meV}\right)$. For the tip, we have considered a $\mathrm{Cu}(111)$ oriented bulk block with a 10 -atom pyramid terminated either with a single metal atom or with a $\mathrm{CO}$ adsorbed underneath it (see sketches at the right in Fig. 5). We will refer to them hereafter as the clean or $\mathrm{Cu}$ tip and the CO tip, respectively.

Among the SIESTA related parameters, the choice of the AO basis set is the most problematic one since there is no systematic yet efficient scheme to converge the total energy with the basis set. ${ }^{41,42}$ In our case, we always employ the so-called double-zeta polarized (DZP) basis set which is known to yield a rather accurate description of the valence states (we will not address here the parameters involved in the pseudopotential generation). However, we have found it highly relevant for the reliability of the entire formalism to employ very extended AOs for all surface and apex atoms. Accordingly, we set confinement energies of $E_{c}=100 \mathrm{meV}$ in the basis generation process if the atoms are contained in any bulk PL and reduce it to $E_{c}=10 \mathrm{meV}$ if they belong to the apex or the sample surface PLs. This way, the spatial extension of the electronic density in the vacuum region is largely extended, thus reproducing better the expected exponential decay of the current with the tip-sample normal distance $z_{\text {tip. }}$. Furthermore, their use systematically lowers the total energies in the slab calculations, which is indicative of a higher accuracy as the total energy is variational with the basis set.

Figures 5(a) and 5(c) show $\log _{10} I\left(z_{\text {tip }}\right)$ plots simulated for the $\mathrm{Cu}$ and $\mathrm{CO}$ tips, respectively, placing the apex on top of a $\mathrm{CO}$ molecule (blue lines) and on top of the $\mathrm{Cu}(111)$ substrate (green); only the elastic contribution to the current is considered here. The plots show a fairly linear behavior in the 5- to 8- $\AA$ interval. Beyond this distance, the exponential decay increases considerably due to the strict localization of the AOs. Still, the linear interval covers the $10^{-1}-10^{1} \mathrm{nA}$ range in all cases, which corresponds to the usual tunneling currents used in STM experiments. The satisfying part is that the contrast inversion experimentally observed for the adsorbed $\mathrm{CO}$ upon transfer of a $\mathrm{CO}$ to the tip is well reproduced; ${ }^{40}$ for the clean tip, the current is slightly larger over the substrate than over the $\mathrm{CO}$ (green line above blue), while the situation is reversed for the $\mathrm{CO}$ tip, where the signal on the $\mathrm{CO}$ is significantly larger than on the $\mathrm{Cu}$. Thus, the metallic tip images the $\mathrm{CO}$ as a hole, while for the $\mathrm{CO}$ contaminated one we find a large protrusion.

An alternative way to inspect the validity of the AO basis under TRCs is via the so-called apparent barrier height $(\mathrm{ABH})$, which definition follows from the widely used expression for the STM current as a function of the tip-sample separation $z_{\text {tip }}: I\left(z_{\text {tip }}\right) \propto \exp \left[-\sqrt{\phi}(\mathrm{eV}) z_{\text {tip }}(\AA)\right]$. Here, $\phi$ is the $\mathrm{ABH}$ and should take values around the metals work functions; that is, $\phi=3-5$ eV. In Figs. 5(b) and 5(d) we plot the ABHs following the same color scheme as in Figs. 5(a) and 5(c). The CO tip on top of the $\mathrm{CO}$ [blue line in Fig. 5(d)] is the only case where the $\mathrm{ABH}$ diminishes as the tip is retracted up to $8 \AA$. For the rest of the situations, the ABHs increase with increasing $z_{\text {tip }}$ whereas one would expect a saturation value at around the work function, averaged between the two electrodes. The ABHs tend to be overestimated, particularly for the CO tip, where values larger than $10 \mathrm{eV}$ are already found at realistic distances of around $7 \AA$. Thus, the AO localization acts as an additional artificial potential barrier to the tunneling electrons. The use of a confinement energy as small as $E_{c}=10 \mathrm{meV}$ is therefore still not enough to provide accurate $I\left(z_{\text {tip }}\right)$ curves and, hence, any calculated tip-sample distances or the actual topographies should be considered only as semiquantitative. Anyhow, we note that the use of more localized basis set, as is done in most SIESTA related studies, may even yield results which are qualitatively wrong. This is demonstrated by the dashed lines in Fig. 5, which correspond to analogous calculations after setting $E_{c}=100 \mathrm{meV}$ for the generation of all AOs in the system. Now, the logarithmic curves deviate from linearity already at $6 \AA$ (on $\mathrm{CO}$ ) while the ABHs reach unrealistic values well above $30 \mathrm{eV}$. Even worse, this basis set would wrongly predict a bump for the CO.

Other relevant parameters related to the DFT calculations are the choice of the exchange-correlation (XC) functional, the $k$-sampling mesh, and the real-space grid employed to evaluate numerically three-center integrals, the XC energy density, or to solve the Poisson equation. To remain consistent, we perform all calculations under the generalized gradient approximation (GGA) scheme. ${ }^{43}$ On the other hand, the total energy can be converged against the reciprocal and real-space meshes, although the required fineness of the grids is system dependent. 
For the present $\mathrm{Cu}+\mathrm{CO}$ system we used a real-space mesh resolution of $0.07 \AA$ (or $500 \mathrm{Ryd})$, while a $(16 \times 16) k$ supercell relative to the bulk $\mathrm{Cu}(111)$ was used in reciprocal space.

A crucial point for an accurate IETS simulation is the correct evaluation of the actual VMs. However, the calculation of the vibrational spectra according to the standard formalism outlined in Sec. III B presents at least three main sources of error: (i) neglect of anharmonic effects, (ii) a poor description of the metal atoms vibrations since phonons are essentially ignored, and (iii) the accuracy in the numerical evaluation of the force derivatives. In general, these errors become larger as the energy of the VM decreases. After extensive tests, we have verified that employing shifts around $\delta r= \pm 0.05 \AA$ in the numerical evaluation of the atomic force derivatives yield reliable values for VMs with frequencies above $10 \mathrm{meV}$. However, for smaller energies, the reliability of the calculated vibrational states is dubious and the associated low-frequency spectra should be considered with care. A clear proof of this inaccuracy are the imaginary frequencies often obtained ${ }^{20}$ or the fact that the lowest-frequency spectra are not robust against the number of $\mathrm{Cu}$ atoms included in the dynamic region.

We now discuss the parameters related to the Green's functions. For the present $\mathrm{Cu}(111)+\mathrm{CO}$ system, the surface PL $s$ comprised the $\mathrm{CO}$ molecule plus the first three $\mathrm{Cu}$ layers, while for the slab calculation in Fig. 4(f) up to $10 \mathrm{Cu}$ layers were included. In order to ensure that self-consistency is preserved in the construction of the semi-infinite surface, ${ }^{37}$ we align the vacuum levels of the bulk and slab calculations by comparing the onsite energies of the atoms in the former case with those at the center of the slab, in particular, those at the fifth layer. The vacuum level for the bulk calculation is then shifted accordingly while the accuracy of the self-consistent procedure may be checked by inspecting the deviations between the onsite energies after the shift. Here, the maximum deviation was just $13 \mathrm{meV}$ for a $739-\mathrm{meV}$ shift. The same procedure is followed for the tip semi-infinite block, for which we included seven $\mathrm{Cu}(111)$ bulk layers in the slab calculation of Fig. 4(d) and compared the onsite energies at the fourth layer with the bulk ones for the vacuum level alignment to obtain a shift of $626 \mathrm{meV}$ and maximum deviation of $22 \mathrm{meV}$.

On the other hand, in the actual Green's function calculation, a finite imaginary part of the energy $E_{i}$ replaces the $0^{+}$limit in Eq. (37). This leads to an extra broadening of the peaks in the density of states (DOS) as well as to a damping of the current as it enters the electrodes. ${ }^{31}$ The choice of this value is crucial for computational efficiency purposes when one goes beyond the WBL since (i) small imaginary energies require larger $k$ samplings and (ii) the energy step used in the numerical integration of the differential currents [Eq. (1)] should not exceed $E_{i}$ in order to ensure that no elastic peaks in the sample or tip DOS are skipped. However, too large values may lead to far too small currents (or equivalently, too small tip-sample distances) and therefore should be avoided. Recall that the simulation time per $\vec{r}_{\text {tip }}$ scales linearly with the number of energy points. From extensive tests, values in the range $E_{i}=2-20 \mathrm{meV}$ represent a good compromise.

A closely related parameter is the electronic temperature $k T$ entering the $f_{F D}$ and $f_{B E}$ distributions for electrons and modes, respectively. On one hand, although $k T \geqslant 10 \mathrm{meV}$

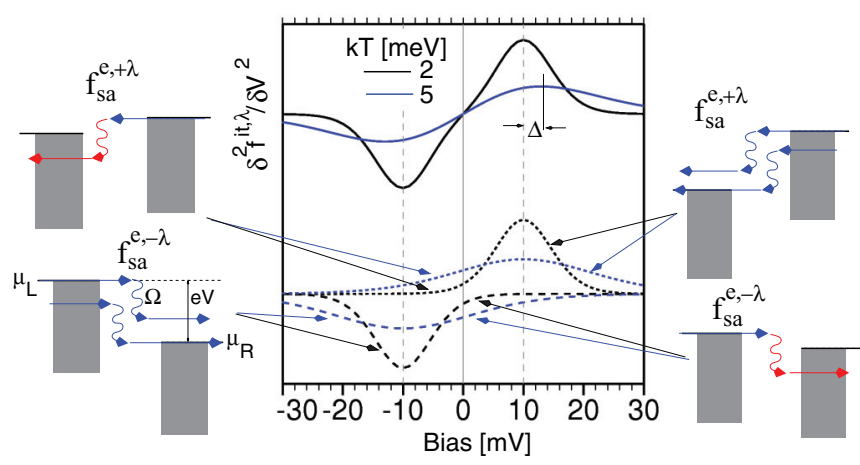

FIG. 6. (Color online) $\partial^{2} f^{i t, \lambda} / \partial V^{2}$ for a single VM with frequency $\Omega=10 \mathrm{meV}$ and for two different temperatures: $k T=2$ (solid dark lines) and $5 \mathrm{meV}$ (solid blue lines). The contributions to $\partial^{2} f^{i t, \lambda} / \partial V^{2}$ arising from the emission factors $f^{e, \pm \lambda}$ are shown at the bottom of the graph with dashed and dotted lines for left and right electron propagation, respectively (see sketches). Red arrows indicate hindered inelastic processes at the lowest temperatures.

smears the electronic structure and eases the convergence in $k$ space, it increases considerably the energy range to be integrated in Eq. (1). On the other hand, $k T$ determines the width of the IETS peaks and, accordingly, a good resolution in the inelastic structure is only achieved for very low values $(k T \leqslant 5 \mathrm{meV})$. In order to address the effect of temperature on the simulated spectra, we consider the LOIT $+\mathrm{WBL}$ expressions (34)-(36), which lead to an inelastic current which is proportional to $f_{\alpha \beta}^{i t, \lambda}$. Since this is the only term where $k T$ enters via the emission and absorption factors $f_{\alpha \beta}^{e / a, \pm \lambda}$, it suffices to analyze the behavior of $\partial^{2} f^{i t, \lambda} / \partial V^{2}$ as a function of temperature to obtain a general picture of its influence on the IETS spectra. This is done in Fig. 6, where we assume a single VM at the surface $\Omega=10 \mathrm{meV}$, and consider two temperatures $k T=2 \mathrm{meV}$ (dark lines) and $5 \mathrm{meV}$ (blue lines). As expected, raising $k T$ increases considerably the peak/dip broadening as inelastic processes in the bias region $|V|<\Omega$, which are forbidden at $k T=0$, start to contribute once the partial occupations of electrons (holes) above (below) the Fermi levels of the electrodes become non-negligible. Since at these temperatures $N^{\lambda}$ is still small, the processes involving emission of a VM, $f^{e, \pm \lambda}$, are still the dominant ones in the spectra (dashed and dotted lines in the figure). In the $|V|<\Omega$ region, the left- and right-propagating currents (dotted and dashed lines, respectively) tend to cancel each other thus inducing an asymmetry in the shape of the peak/dip and, most notably, a small shift $\Delta$ in its maximum/minimum towards larger bias values (in the figure $\Delta \approx 4 \mathrm{meV}$ ). Such shifts may induce erroneous assignments of the lowest-frequency VMs when comparing simulated versus experimental inelastic spectra, implying that the actual temperature is a highly relevant parameter which should be properly taken into account in any IETS analysis.

Next, we address the convergence of the LOIT in $k$ space since it is generally harder to achieve well-converged results in electronic transport calculations than in total energy optimizations or when obtaining the self-consistent Hamiltonian. Figure 7 shows $\left(\partial^{2} I / \partial V^{2}\right) /(I / V)$ spectra for the $\mathrm{Cu}(111)+\mathrm{CO}$ system probed by the $\mathrm{Cu}$ tip for different 
(a) WBL

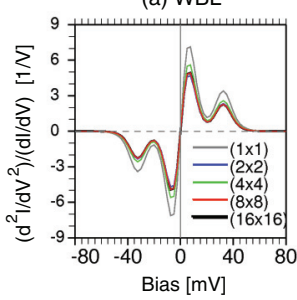

(b) WBL@tip
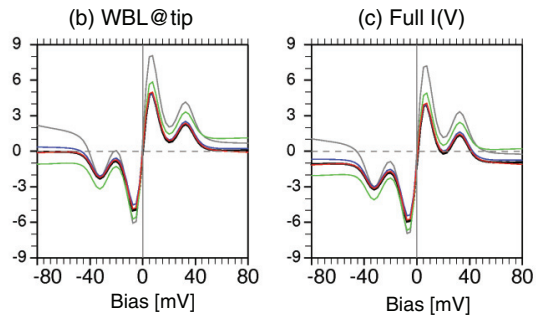

FIG. 7. (Color online) $\left(\partial^{2} I / \partial V^{2}\right) /(I / V)$ curves simulated for the $\mathrm{Cu}$ tip probing a $\mathrm{CO}$ molecule on a $\mathrm{Cu}(111)$ surface under the LOIT approximation and for different $k$ samplings at PL $s$, as indicated in the legend in (a). The $k$ grids refer to the surface $(4 \times 4)$ supercell. The curves have been calculated: (a) assuming the WBL both at the tip and the sample, (b) assuming the WBL only at the tip, and (c) including the entire energy range at the tip and sample. All spectra calculated for $k T=2.5 \mathrm{meV}, E_{i}=20 \mathrm{meV}$, and fixing the $k$ sampling at the tip block to an $(8 \times 8)$ supercell.

LOIT-based approximations and $k$ samplings at the surface $(4 \times 4)$ supercell. The $k$ mesh at the tip block has been fixed to an $(8 \times 8)$ in all cases. The WBL in Fig. 7(a) shows a nice behavior with the $k$ grid, and is well converged for an $(8 \times 8)$ mesh, equivalent to a $(32 \times 32)$ relative to the $(1 \times 1)$ bulk $\mathrm{Cu}(111)$. The curves show a double peak/dip structure corresponding to the $\mathrm{FT}_{\mathrm{CO}}$ and $\mathrm{FR}_{\mathrm{CO}}$ vibrational modes (see next section). The smallest $(1 \times 1)$ grid $(\Gamma$-point calculation) already yields qualitatively correct results, although the inelastic features are overestimated by about $50 \%$. The same picture remains if the WBL is only applied to the tip electronic structure while including the full energy dependence of the scattering states at the sample surface [Fig. 7(b)] or even if the WBL is removed at both sides [Fig. 7(c)]. The resulting spectra and $k$ convergence are very similar to the WBL results, especially for the finest meshes. The main difference is just a sizable downwards shift for the full $I(V)$ case in Fig. 7(c), which arises from the tail of a resonant peak at the tip. Therefore, these results show that (i) the computationally much cheaper WBL approximation is highly reliable and (ii) a good $k$ convergence is perfectly achievable within our formalism. The LOIT + WBL approach is thus ideally suited to simulate IETS spectra. On the other hand, and given the fact that the exact apex geometry and thus its precise electronic structure is in general an experimental unknown, applying the WBL only at the tip seems a promising route to analyze simultaneously the surface LDOS and the IETS.

\section{RESULTS}

The calculation parameters detailed in the previous section led to VM frequencies for the $\mathrm{Cu}(111)-(4 \times 4)+\mathrm{CO}$ system in good agreement with previous works. Namely, out of a total of 23 modes, the most relevant $\mathrm{CO}$ related calculated frequencies are $v_{\mathrm{C}-\mathrm{O}}=246 \mathrm{meV}, v_{\mathrm{CO}-\mathrm{Cu}}=47 \mathrm{meV}, \mathrm{FR}_{\mathrm{CO}}=$ $33 \mathrm{meV}$, and $\mathrm{FT}_{\mathrm{CO}}=2-6 \mathrm{meV}$. Additionally, we also found low-frequency modes involving both the $\mathrm{CO}$ and the $\mathrm{Cu}$ atoms below as if they were a rigid complex, such as stretch modes $v_{\mathrm{COCu}-\mathrm{nCu}}=4 \mathrm{meV}$ or frustrated rotations $\mathrm{FR}_{\mathrm{COCu}}=10 \mathrm{meV}$. For the CO contaminated tip we obtained $33 \mathrm{VMs}$, reflecting the larger number of degrees of freedom arising from the low
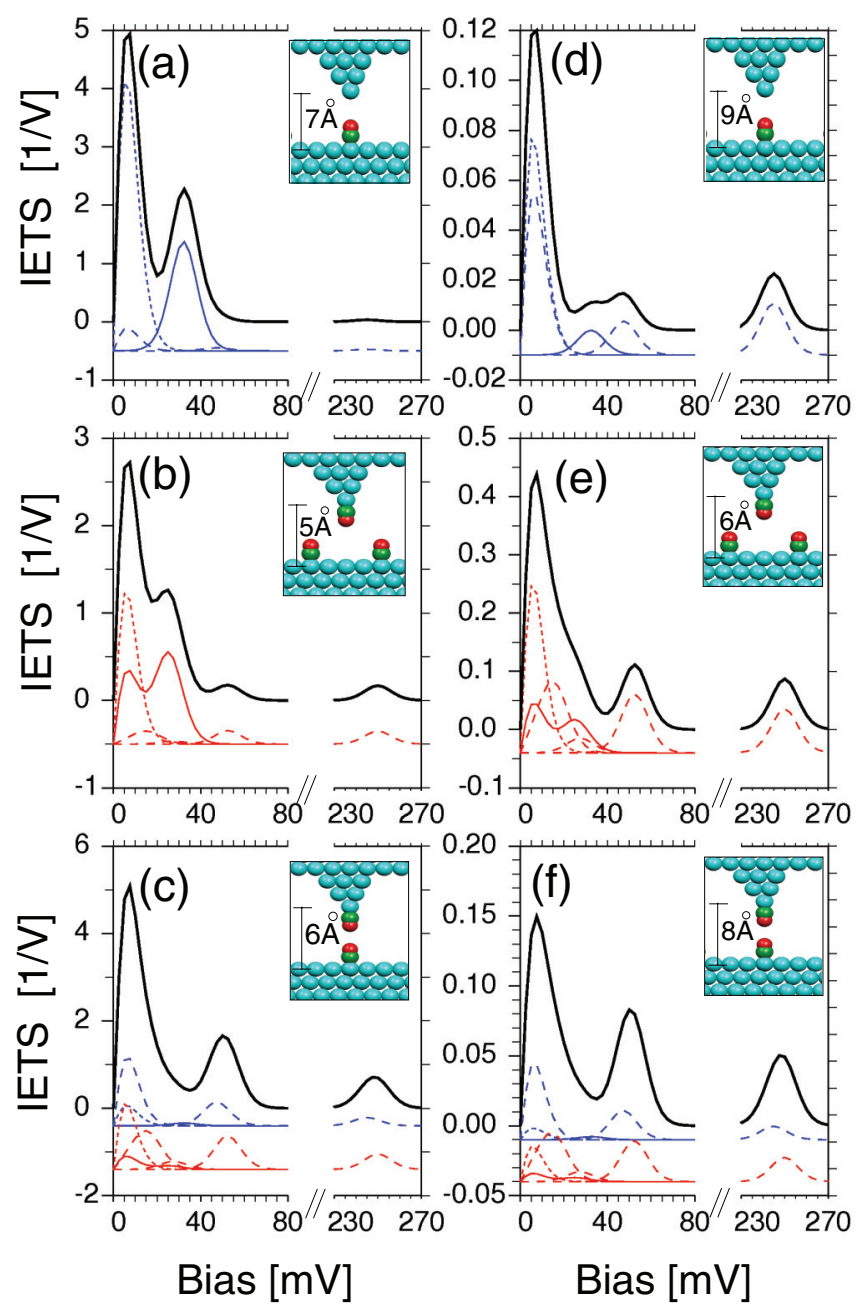

FIG. 8. (Color online) Calculated IETS curves under the LOIT + WBL approximation for the $\mathrm{Cu}(111)+\mathrm{CO}$ system probed by a (a), (d) clean $\mathrm{Cu}$ tip on top of the $\mathrm{CO}$, (b), (e) CO contaminated tip on the $\mathrm{Cu}(111)$ substrate, and (c), (f) $\mathrm{CO}$ contaminated tip on top of the surface CO. Tip-sample distances are indicated in the insets. Solid dark lines: total IETS; dotted, solid, and dashed blue/red lines: contribution arising from the FTs, FRs, and $v$ modes, respectively, with blue for those localized at the surface and red at the $\mathrm{CO}$ terminated tip, offset in $y$ for clarity. All curves calculated under the LOIT + WBL approximation with parameter values: $k T=2.5 \mathrm{meV}$ and $E_{i}=20 \mathrm{meV}$. See text for further explanations.

coordination at the apex. The high-frequency stretch modes are slightly blue-shifted with respect to the previous case, $v_{\mathrm{C}-\mathrm{O}}=$ $251 \mathrm{meV}$ and $v_{\mathrm{CO}-\mathrm{Cu}}=51 \mathrm{meV}$, while the lower-frequency ones tend to red-shift, $\mathrm{FT}_{\mathrm{CO}}=2 \mathrm{meV}, \mathrm{FR}_{\mathrm{CO}}=24-26 \mathrm{meV}$, and/or split due to a large coupling between the molecule and the $\mathrm{Cu}$ atoms motions: $v_{\mathrm{COCu}-\mathrm{nCu}}=14,17$, and $28 \mathrm{meV}$ or $\mathrm{FR}_{\mathrm{COCu}}=3$ and $10 \mathrm{meV}$. However, and as already mentioned in the previous section, the reliability of the calculated lowfrequency modes is scarce.

In Fig. 8, we present normalized IETS curves. $\left(\partial^{2} I / \partial V^{2}\right) /(d I / d V)$, calculated under the LOIT $+\mathrm{WBL}$ approximations for different tip-sample configurations. As sketched in the insets, in Figs. 8(a) and 8(d) we probe the VMs at the surface $\mathrm{CO}$ by considering the clean apex placed 
on top of the molecule; in Figs. 8(b) and 8(e) we probe those at the apex by placing the $\mathrm{CO}$ tip on top of the $\mathrm{Cu}(111)$ substrate; in Figs. 8(c) and 8(f) the same tip is now located above the adsorbed $\mathrm{CO}$ so that we have contributions from the VMs at both the tip and the surface. Dark solid lines give the total IETS signal, while blue and red ones refer to the contributions from different types of modes localized at the surface and tip, respectively; recall that the LOIT expression (43) directly provides the VM resolved contributions. We use dotted lines for the sum over FT modes, solid for FRs and dashed for the stretch modes, although we warn that the assignment of some low-frequency modes to one type or the other is often ambiguous. The graphs at the left, Figs. 8(a)-8(c), are calculated for tip-sample distances $z_{\text {tip }}$ within the range for which the ABHs take reasonable values (see Fig. 5). In this range, the magnitude of the IETS signal remains fairly constant between $3-10 \mathrm{~V}^{-1}$ as expected after the normalization by $(d I / d V)$. In the graphs at the right, Figs. 8(d)-8(f), the tip is retracted by $1-2 \AA$, thus falling into the range where the ABHs attain too large values while the IETS peaks are an order of magnitude smaller and, hence, the results are less accurate. Still, we show them since they reveal an interesting trend which will be discussed below.

When the clean tip is on top of the CO [Fig. 8(a)], we find a large signal for the low-frequency FTs and the FRs with hardly any contribution from the stretch modes. The resulting IETS curve is a well-defined double-peak structure which is in line with previous experimental ${ }^{13,14}$ and theoretical ${ }^{14,34,35}$ studies for the same system. However, our FT contribution seems to be overestimated with respect to these works, where a similar height was found for the FT and FR modes. We ascribe this discrepancy to the inaccuracy associated to the low-frequency modes, as mentioned above. A similar picture holds when the CO tip is placed on top of the substrate [Fig. 8(b)]; we only present the contributions from the VMs localized at the apex since those localized at the surface, although included in the calculation, give negligible intensities. Again, the FT and FR modes dominate the spectra resulting in a double-peak structure although this time that associated to the $\mathrm{FR}_{\mathrm{CO}}$ mode, given its smaller frequency, overlaps more strongly with the larger peak at the lowest frequency and appears as a shoulder of the latter. The stretch modes, on the other hand, yield a sizable signal so that the high-frequency $v_{\mathrm{C}-\mathrm{O}}$ and $v_{\mathrm{CO}-\mathrm{Cu}}$ peaks can be resolved in the total IETS. Finally, in Fig. 8(c) vibrations both at the tip and the surface (56 VMs in total) are considered by placing the CO tip on top of the adsorbed CO. Surprisingly, the stretch modes now yield a large signal becoming the dominant ones throughout the spectra, while the contribution from the FR modes is strongly reduced. Notice, for instance, the large peaks at around 4 and $15 \mathrm{meV}$ associated to the $v_{\mathrm{COCu}-\mathrm{nCu}}$ stretch modes at the surface and tip, respectively. Curiously, the contribution of each type of modes is quite similar either if they are localized at the surface or the tip. Given the thermal broadening used in the simulations, $k T=2.5 \mathrm{meV}$, one can not resolve in the total IETS among vibrations at the surface or the tip as the $v_{\mathrm{C}-\mathrm{O}}$ and $v_{\mathrm{CO}-\mathrm{Cu}}$ modes merge into single broad peaks. The absence of hardly any inelastic activity from the FR modes is at contrast with the work of Ref. 20, where they still found the FR mode as the most relevant one after considering a $\mathrm{CO}$ terminated tip, although an increase of the

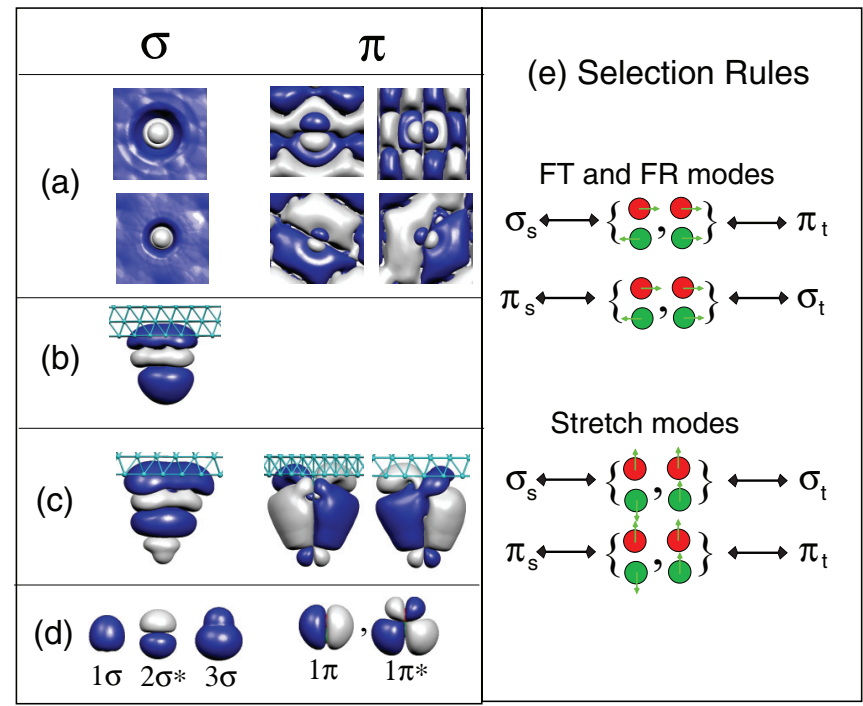

FIG. 9. (Color online) (a) Top views of isosurfaces for several scattering states $\left|a_{s}^{s, m_{s}}\right\rangle$ at the $\mathrm{Cu}(111)+\mathrm{CO}$ surface grouped according to their symmetry character. The $\mathrm{CO}$ is always located at the center of the image, with white and blue colors used for positive and negative isovalues, respectively. (b) Side view of an isosurface corresponding to the most relevant scattering state $\left|a_{a}^{s, m_{a}}\right\rangle$ at the $\mathrm{Cu}$ tip. The atoms drawn at the top belong to the tip $\mathrm{Cu}(111)$ electrode. (c) Same as (b) but for the CO tip, with one $\sigma$ and two $\pi$ states. (d) Side views of the CO MOs which provide the largest contribution to the inelastic currents. (e) Diagram showing the relevant tunneling paths for the FT, FR, and stretch modes according to the IETS propensity rules.

$v_{\text {CO-Cu }}$ signal for this particular tip was also reported. The reason for this discrepancy may be due to the more contracted AOs employed in that work or the actual tip modeling, as the $\mathrm{CO}$ was adsorbed below a $\mathrm{Cu}(111)$ surface instead of the sharp pyramid considered here.

\section{A. Propensity rules}

IETS propensity rules have been the subject of several works. ${ }^{24,27,28,34,45}$ A particularly simple derivation of such rules was presented by Paulsson et al. in Ref. 34 employing a Fermi golden rule expression for the phonon emission rate $\gamma_{\lambda}^{\mathrm{FGR}}=(e / \pi \hbar) \operatorname{Tr}\left[M_{s a}^{\lambda} A_{a a} M_{a s}^{\lambda} A_{s s}\right]$, and has been later confirmed and analyzed in more detail precisely for the $\mathrm{Cu}(111)+\mathrm{CO}$ system. ${ }^{35}$ The propensity rules were found to follow simple symmetry arguments relating the $\sigma / \pi$ character of the surface and tip propagating states to the actual VM type; $\sigma(\pi)$ refers to symmetric (antisymmetric) states relative to twofold rotations about the surface normal direction. In short, if one assigns a $\pi$ character to the FR and FT vibrations and a $\sigma$ character to the stretch modes, then the dominant inelastic paths are those for which the combined symmetry of the surface and tip states together with that of the VM is of $\sigma$ type [see Fig. 9(e)]. That is, if for a given scattering path coupling states at the tip apex $a$ and sample $s$ with $\lambda$ we denote their respective symmetries $\tau$ as $\left(\tau_{s}, \tau_{\lambda}, \tau_{a}\right)$, then combinations such as $\left(\sigma_{s}, \sigma_{\lambda}, \sigma_{a}\right),\left(\pi_{s}, \pi_{\lambda}, \sigma_{a}\right)$, or $\left(\pi_{s}, \sigma_{\lambda}, \pi_{a}\right)$ will be large compared to odd combinations such as $\left(\sigma_{s}, \sigma_{\lambda}, \pi_{a}\right)$ or $\left(\pi_{s}, \pi_{\lambda}, \pi_{a}\right)$. Paulsson et al. proposed to transform the 
TABLE I. Percentual contributions of different tunneling paths to the elastic current and inelastic currents associated to different types of modes calculated under the three configurations depicted in Figs. 8(a)-8(c). Values in parentheses correspond to the same cases but after retracting the tip by $1-2 \AA$ [Figs. 8(d)-8(f)].

\begin{tabular}{llcccc}
\hline \hline & $\mathrm{VM}$ & $\left(\sigma_{s}, \sigma_{a}\right)$ & $\left(\sigma_{s}, \pi_{a}\right)$ & $\left(\pi_{s}, \sigma_{a}\right)$ & $\left(\pi_{s}, \pi_{a}\right)$ \\
\hline \multirow{4}{*}{ Cu tip } & Elastic & $78(85)$ & $0(0)$ & $0(0)$ & $20(13)$ \\
on CO & $\mathrm{FT}(s)$ & $0(1)$ & $0(0)$ & $98(97)$ & $0(0)$ \\
& $\mathrm{FR}(s)$ & $0(0)$ & $0(1)$ & $98(97)$ & $0(0)$ \\
& $v(s)$ & $86(95)$ & $0(0)$ & $1(0)$ & $12(4)$ \\
& Elastic & $57(11)$ & $1(0)$ & $3(2)$ & $37(85)$ \\
CO tip & $\mathrm{FT}(a)$ & $0(0)$ & $85(73)$ & $2(3)$ & $11(22)$ \\
on Cu & $\mathrm{FR}(a)$ & $0(0)$ & $86(72)$ & $1(7)$ & $11(19)$ \\
& $v(a)$ & $4(27)$ & $0(1)$ & $1(4)$ & $93(67)$ \\
& $\operatorname{Elastic}$ & $2(0)$ & $0(0)$ & $1(1)$ & $96(98)$ \\
& $\mathrm{FT}(s)$ & $0(0)$ & $69(66)$ & $27(28)$ & $2(5)$ \\
CO tip & $\mathrm{FT}(a)$ & $0(0)$ & $73(53)$ & $25(45)$ & $0(0)$ \\
on CO & $\mathrm{FR}(s)$ & $0(1)$ & $78(91)$ & $20(7)$ & $0(0)$ \\
& $\mathrm{FR}(a)$ & $0(0)$ & $71(39)$ & $27(59)$ & $1(1)$ \\
& $v(s)$ & $3(4)$ & $0(0)$ & $1(0)$ & $95(94)$ \\
& $v(a)$ & $2(3)$ & $0(0)$ & $1(1)$ & $96(95)$ \\
\hline \hline
\end{tabular}

spectral matrices $A_{\alpha \alpha}$ into the eigenchannels basis projected at the surface and the tip as they represent a natural basis for the propagating states at each side of the junction and ease the derivation of the propensity rules. Indeed, it was found sufficient to include just a small number of eigenchannels in order to obtain a good estimate of $\gamma_{\lambda}^{\mathrm{FGR}}$.

Our LOIT approach is specially suited for performing a similar analysis of the propensity rules, albeit it offers several advantages. We first recall that Eq. (43) for the inelastic current implies that the height of a given IETS peak associated to VM $\lambda$ is determined by the $\left|\left\langle a_{s}^{s, m_{s}}\left|M_{s a}^{\lambda}\right| a_{a}^{s, m_{a}, \pm \lambda}\right\rangle\right|^{2}$ terms. Indeed, this equation is highly reminiscent of the $\gamma_{\lambda}^{\mathrm{FGR}}$ expression of Paulsson et al., except that the former exploits the TRCs replacing the eigenchannel projections by the surface scattering states $\left|a_{\alpha}^{s, m_{\alpha}}\right\rangle$. Hence, the symmetry of these states can be examined independently and prior to the calculation of the full system Green's function (or the eigenchannels). We have found that for the $\mathrm{Cu}+\mathrm{CO}$ surface, around 10 states are enough to accurately compute the inelastic currents. In Fig. 9(a), we plot top views of isosurfaces corresponding to some representative states grouped according to their $\tau_{s}$ symmetry. At the $\mathrm{Cu}$ tip, on the other hand, just one $\sigma$ state dominates by far all elastic and inelastic currents [see side view in Fig. 9(b)] while, at the CO tip, only one $\sigma$ and two $\pi$ channels are necessary [see Fig. 9(c)]. In Table I, we provide the percentual contribution to the elastic $\left|\left\langle a_{s}^{s, m_{s}}\left|F_{s a}\right| a_{a}^{s, m_{a}, \pm \lambda}\right\rangle\right|^{2}$ and inelastic $\left|\left\langle a_{a}^{s, m_{a}}\left|M_{s a}^{\lambda}\right| a_{a}^{s, m_{a}, \pm \lambda}\right\rangle\right|^{2}$ rates associated to the FT, FR, and $v$ modes arising from tunneling paths involving these states and for the same configurations as those depicted in Fig. 8. The fulfillment of the propensity rules is quite remarkable and applies to all cases regardless the VMs are located at the apex or the surface or the actual tip-sample distance. FT and FRs are always largely dominated by $\left(\pi_{s}, \sigma_{a}\right)$ and $\left(\sigma_{s}, \pi_{a}\right)$ paths, whereas stretch modes mainly interact with $\left(\sigma_{s}, \sigma_{a}\right)$ paths for the $\mathrm{Cu}$ tip and $\left(\pi_{s}, \pi_{a}\right)$ for the $\mathrm{CO}$ tip. Notice, on the other hand, that the elastic rates are dominated by paths where surface and tip states share the same symmetry.

We may also attempt an even simpler derivation of the propensity rules following a similar approach to that of Nakamura et al $^{27}$ To this end, we consider the CO tip placed on top of the CO [Figs. 8(c) and 8(f)] and transform the $M_{s a}^{\lambda}$ matrix elements linking the COs at the surface and the apex from the $\mathrm{AO}$ basis set to the molecular orbitals (MOs): $\widetilde{M}_{s a}^{\lambda}=$ $V_{s}^{\dagger} M_{s a}^{\lambda} V_{a}$, where $V_{s / a}$ holds the MOs (in our case those of the $\mathrm{CO}$ at the surface and the tip), and $\widetilde{M}_{s a}^{\lambda}$ stands for the $e$-VM coupling in the new basis. Recall that this new basis is a mixture of $\mathrm{AO}$ and MOs since all the terms related to the metal atoms are still described by the former. The MOs are trivially obtained by performing a self-consistent calculation of the isolated $\mathrm{CO}$ keeping its geometry to that obtained for the relaxed surface and CO tip. Next, by applying the MO transforms to the scattering states $\left|\widetilde{a}_{\alpha}^{s, m_{\alpha}}\right\rangle=V_{\alpha}^{-1}\left|a_{\alpha}^{s, m_{\alpha}}\right\rangle$, one may evaluate Eq. (43) in this mixed basis and extract the contribution of all the scattering paths for a given VM. It turns out that out of a total of around 900 orbitals used to describe the STM interface, just $5 \mathrm{CO}$ MOs dominate by far both the elastic and inelastic currents. They are sketched in Fig. 9(d) again grouped by their symmetry character. Within the $\sigma$ group, the $3 \sigma$ MO generally provides by far the largest contribution followed by the $1 \sigma$, while the $2 \sigma^{*}$ tends to yield a considerable contribution but of negative sign arising from strong interference effects. Within the $\pi$ group, the $1 \pi^{*} \mathrm{MO}$ is typically the dominant state and next the $1 \pi$. The percentual contributions to the elastic and inelastic currents associated to the FT, FR, and $v$ modes arising from $\left(\tau_{s}, \tau_{t}\right)$ tunneling paths involving only these MOs are very similar, within $10 \%$, to the values given in Table I, thus again corroborating the propensity rules. Unfortunately, such a simple MO approach is only useful whenever the main tunneling paths involve molecules at both the tip and the sample. For the other cases, $\mathrm{Cu}$ tip on $\mathrm{CO}$ or $\mathrm{CO}$ tip on $\mathrm{Cu}$, the large hybridization among the metal atom AOs leads to interference effects which complicate the symmetry analysis.

\section{B. IETS dependence on tip-sample distance}

Several works have addressed the evolution of the VM features in energy and height from the tunneling to contact regimes. ${ }^{14,44}$ These studies showed that the contribution of the inelastic processes tends to increase the total current in the former regime, which is in accordance with our LOIT approach where expression (43) has the same sign as its elastic counterpart (42). However, in those studies the AO basis employed was rather localized and therefore, as shown in Sec. IV, the calculated currents at large $z_{\text {tip }}$ values can not be trusted. The use of extended AOs allows a more accurate insight into the dependence of the IETS on $z_{\text {tip }}$ under TRCs. Indeed, comparing Figs. 8(a) and 8(b) versus 8(d) and 8(e) reveals a strong increase of the stretch modes peak heights relative to the FR and FT ones as the tip is retracted by $1-2 \AA$. As a result, the calculated IETS curves may even differ qualitatively.

In Fig. 10, we plot the evolution of the IETS peak heights as a function of $z_{\text {tip }}$. Despite the normalization $d I / d V$ factor, there is an overall decrease of the IETS intensities with increasing tip-sample distance indicating that the it couplings 

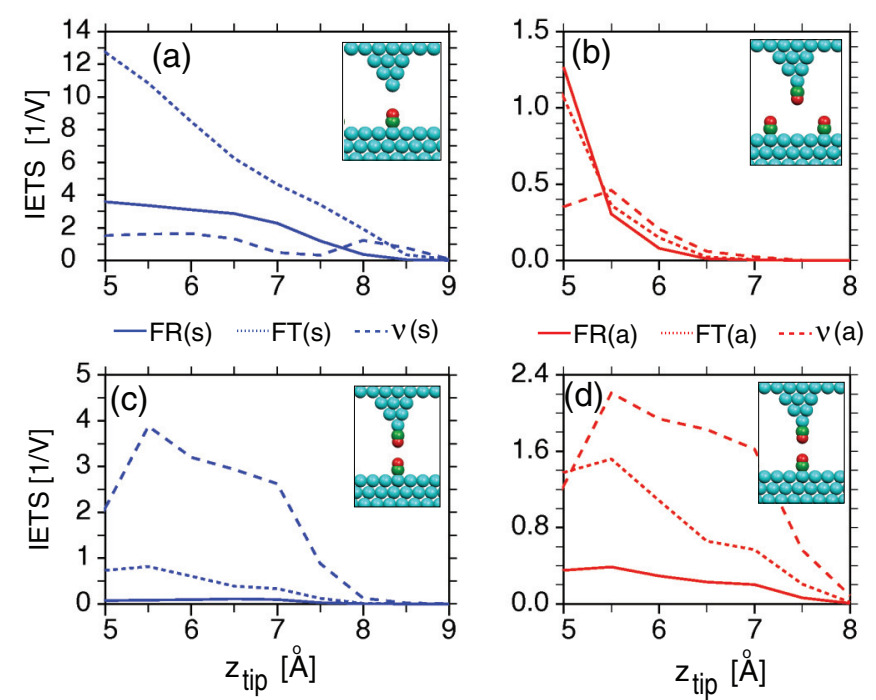

FIG. 10. (Color online) Evolution of the IETS peaks with $z_{\text {tip }}$ for (a) $\mathrm{Cu}$ tip on $\mathrm{CO}$, (b) $\mathrm{CO}$ tip on $\mathrm{Cu}$, (c), (d) CO tip on CO. Solid, dashed, and dotted lines correspond to the sum of the IETS peaks associated to FRs, FTs, and stretch modes, respectively, while blue (red) lines refer to VMs located at the surface (tip).

decay faster into vacuum than the elastic ones. The figure also reveals different decay factors for each type of mode and tip. For the $\mathrm{Cu}$ tip on $\mathrm{CO}$ case shown in Fig. 8(a), the FTs dominate over the FRs and next the $v$ modes, with a larger decay for the former. Curiously, the stretch modes contribution shows a sudden increase at $z_{\text {tip }}=8.5 \AA$ and becomes even larger than that of the FTs, hence leading to qualitatively different IETS. We ascribe this increase to an optimum overlap at this distance between the $\sigma$ state at the $\mathrm{Cu}$ tip and those at the surface which maximizes the $e$-VM coupling. The fact that, to our knowledge, the interchange in the peak heights between the the FT and $v$ modes has not been reported experimentally is most probably related to a small signal-to-noise ratio at such large distances (or small currents), which hinders the resolution of any IETS peaks.

For the CO tip, the stretch modes are always the dominant ones, except at the closest distances $z_{\text {tip }} \leqslant 5 \AA$, where we find a sudden decrease in their contribution and may even become smaller than the rest of modes as is the case when the tip is on top of the $\mathrm{Cu}$ [Fig. 10(b)]. This is the reason for the different aspect of the spectra between Figs. 5(b) and 5(e). At larger distances, all apex modes decay in a similar way, with the FR modes providing the smallest contribution. When the same tip is placed on top of the $\mathrm{CO}$, we find a relatively similar behavior between the modes located at the surface [see Fig. 10(c)] and those at the apex [see Fig. 10(d)]. This time, all IETS heights decay more smoothly except the stretch ones, which at $z_{\text {tip }} \geqslant 7 \AA$ present a sudden increase in the slope albeit not big enough to change the hierarchy among the types of modes or, equivalently, the shape of the IETS curves [see Figs. 3(c) versus 3(f)].

The above results suggest that the tip-sample distance may result in a crucial parameter in determining the shape of the inelastic spectra, particularly at small distances, but also, for the $\mathrm{Cu}$ tip case, at large $z_{\text {tip }}$ values. However, since curves (d)-(f) in Fig. 5 have been calculated at large tip-sample distances for which our AO basis set is not too accurate, further tests with even more extended states seem necessary to confirm this effect.

\section{CONCLUSIONS}

We have revisited the SCBA formalism within the context of IETS simulations under an STM setup. As long as the system is under TRCs and the molecules are chemisorbed either at the surface or the tip or both, the leading inelastic events are found to be those involving a single VM-mediated tunneling event, while any intralead inelastic scattering may be safely discarded. This leads to a particularly simple, yet accurate, Fermi golden rule type expression for the inelastic current [Eq. (31)], which we denote as the lowest order in inelastic tunneling (LOIT) approximation. Furthermore, in the case of physisorbed molecules, the LOIT + ILSCBA approach, which treats the intralead inelastic scattering events within each electrode self-consistently, is found to perform as accurately as the full SCBA approach as long as the system remains in a tunneling regime.

The LOIT allows a drastic reduction of the computational cost since first the structural optimization and next the vibrational spectra can be calculated independently for the surface and the tip. We have implemented the LOIT approximation within the GREEN package, ${ }^{31}$ which is specially designed for STM simulations treating the surface and the tip on equal footings despite the complexity of the system [see Fig. 1(a)]. Two relevant geometrical features of the implementation, not generally accounted for in related codes, are the fact that the sample and tip electrodes may differ in geometry and nature as well as an efficient $k$-sampling scheme at both electrodes, while the tip apex is described as a nonperiodic cluster stacked below the tip electrode. The simulations are carried out at the $a b$ initio level via a suitable interface to the DFT-based SIESTA (Ref. 33) code, whereby the Hamiltonian of the entire system is calculated by pieces (sample and tip bulk electrodes, surface, and apex) and next assembled via Green's function techniques. Self-consistency is fully preserved at all blocks except at the apex, for which the matrix elements $H_{a a}, H_{a s}$, or $M_{s a}^{\lambda}$ are calculated under different geometries than those used in the IETS simulation; in the former cases, we use a 2D supercell [e.g., a $(4 \times 4)$ ], while in the latter the apex is assumed isolated. This approximation is anyhow necessary to properly describe the $k$ mixing at the apex, while its accuracy can be tested by increasing the size and the number of atoms in the 2D supercell.

The implementation is efficient enough to allow the study in detail of the numerous parameters that enter the actual calculation. We have performed such an analysis for the widely studied $\mathrm{Cu}(111)+\mathrm{CO}$ system under a clean and a $\mathrm{CO}$ functionalized tip in order to identify and quantify the main sources of inaccuracy associated to them. In the first place, we have evidenced the need of highly extended AOs as basis set in order to reproduce reasonably well the TRCs. Second, it turns out that a good $k$ convergence in the $\partial^{2} I / \partial V^{2}$ spectra can be achieved with moderate $k$ samplings, while the usual $\Gamma$-point approximation provides qualitatively correct results although it tends to overestimate the inelastic peak/dip heights. Next, assuming WBLs at both electrodes 
is proven to be highly reliable for this system, with the valuable advantage that it involves much lighter calculations. The calculated inelastic spectra for the $\mathrm{Cu}$ tip shows two main peaks associated to the FT and FR modes, in good accordance with previous experimental and theoretical works. On the other hand, under the $\mathrm{CO}$ tip, the intensity of the FR modes decreases considerably and the stretch modes become the dominant ones.

Finally, the LOIT expressions provide a natural way to derive the propensity rules for the appearance or not of inelastic features associated to a particular VM. The symmetry of the surface scattering states can be examined independently and prior to the calculation of the full system, thus allowing us to predict which inelastic paths should be the most relevant ones for each VM in virtue of the propensity rules. This could be useful, for instance, to select appropriate tips depending on the type of vibrations present in an IETS experiment.

To conclude, we mention the potential application of the LOIT approach to simulate IETS maps at selected voltages. Such calculations are not yet feasible mainly due to the large computational cost associated to the simulation, at the ab initio level, of a topographic image even if only the elastic current is considered. In addition to the typically large number of pixels required in an image, adjusting the tip-sample distance to yield a preselected current value at a given pixel requires several slab calculations of the type shown in Fig. 4(f), each taking a few tens of hours in standard multicore platforms. An alternative route is to evaluate the $H_{s a}$ and $M_{s a}^{\lambda}$ matrices over an ample set of $\vec{r}_{\text {tip }}$ tip positions and use them as data to set up a parametrization scheme that allows us to approximate these matrices on the fly for any $\vec{r}_{\text {tip }}$. Such an approach has already been implemented for the $H_{s a}$ matrix elements when simulating topographic images under the elastic regime, ${ }^{46}$ while extending it to the $M_{s a}^{\lambda}$ matrices in order to generate IETS maps in a fast way will be the subject of future works.

\section{ACKNOWLEDGMENT}

J.C. acknowledges financial support from the Spanish Ministry of Innovation and Science under Contract No. MAT2010-18432. *erwin.rossen@gmail.com

†c.f.j.flipse@tue.nl

¥jcerda@icmm.csic.es

${ }^{1}$ A. H. Flood, J. F. Stoddart, D. W. Steuerman, and J. R. Heath, Science 306, 2055 (2004).

${ }^{2}$ A. Troisi and M. Ratner, Small 2, 172 (2006).

${ }^{3}$ J. G. Kushmerick, J. Lazorcik, C. H. Patterson, R. Sashidhar, D. S. Seferos, and G. C. Bazan, Nano Lett. 4, 639 (2004).

${ }^{4}$ M. A. Reed, C. Zhou, C. J. Muller, T. P. Burgin, and J. M. Tour, Science 278, 252 (1997).

${ }^{5}$ D. Djukic, K. S. Thygesen, C. Untiedt, R. H. M. Smit, K. W. Jacobsen, and J. M. van Ruitenbeek, Phys. Rev. B 71, 161402(R) (2005).

${ }^{6}$ H. Ueba, Surf. Rev. Lett. 10, 771 (2003).

${ }^{7}$ M. Galperin, M. A. Ratner, and A. Nitzan, J. Phys.: Condens. Matter 19, 103201 (2007).

${ }^{8}$ K. J. Franke and J. I. Pascual, J. Phys.: Condens. Matter 24, 394002 (2012).

${ }^{9}$ N. Okabayashi, Y. Konda, and T. Komeda, Phys. Rev. Lett. 100, 217801 (2008).

${ }^{10}$ C. M. Whelan, C. J. Barnes, C. G. H. Walker, and N. M. D. Brown, Surf. Sci. 425, 195 (1999).

${ }^{11}$ J. R. Hahn and W. Ho, Phys. Rev. Lett. 87, 196102 (2001).

${ }^{12}$ B. C. Stipe, M. A. Rezaei, and W. Ho, Science 280, 1732 (1998).

${ }^{13}$ A. J. Heinrich, C. P. Lutz, J. A. Gupta, and D. M. Eigler, Science 298, 1381 (2002).

${ }^{14}$ L. Vitali, R. Ohmann, K. Kern, A. Garcia-Lekue, T. Frederiksen, D. Sánchez-Portal, and A. Arnau, Nano Lett. 10, 657 (2010).

${ }^{15}$ A. Mugarza, R. Robles, C. Krull, R. Korytar, N. Lorente, and P. Gambardella, Phys. Rev. B 85, 155437 (2012).

${ }^{16}$ N. Mingo and K. Makoshi, Phys. Rev. Lett. 84, 3694 (2000).

${ }^{17}$ N. Lorente and M. Persson, Phys. Rev. Lett. 85, 2997 (2000).

${ }^{18}$ U. Harbola, J. Maddox, and S. Mukamel, Phys. Rev. B 73, 205404 (2006).

${ }^{19}$ H. Ren, J. Yang, and Y. Luo, J. Chem. Phys. 130, 134707 (2009).

${ }^{20}$ T. Frederiksen, M. Paulsson, M. Brandbyge, and A.-P. Jauho, Phys. Rev. B 75, 205413 (2007).
${ }^{21}$ Y. Asai, Phys. Rev. Lett. 93, 246102 (2004); T. Shimazaki and Y. Asai, Phys. Rev. B 77, 115428 (2008).

${ }^{22}$ M. Galperin, M. A. Ratner, and A. Nitzan, J. Chem. Phys. 121, 11965 (2004).

${ }^{23}$ G. C. Solomon, A. Gagliardi, A. Pecchia, T. Frauenheim, A. Di Carlo, J. R. Reimers, and N. S. Hush, J. Chem. Phys. 124, 94704 (2006).

${ }^{24}$ A. Gagliardi, G. C. Solomon, A. Pecchia, T. Frauenheim, A. Di Carlo, N. S. Hush, and J. R. Reimers, Phys. Rev. B 75, 174306 (2007).

${ }^{25}$ T. Mii, S. G. Tikhodeev, and H. Ueba, Phys. Rev. B 68, 205406 (2003); H. Ueba, T. Mii, and S. G. Tikhodeev, Surf. Sci. 601, 5220 (2007).

${ }^{26}$ M. Paulsson, T. Frederiksen, and M. Brandbyge, Phys. Rev. B 72, 201101(R) (2005).

${ }^{27}$ H. Nakamura, K. Yamashita, A. R. Rocha, and S. Sanvito, Phys. Rev. B 78, 235420 (2008).

${ }^{28}$ A. Troisi and M. A. Ratner, J. Chem. Phys. 125, 214709 (2006).

${ }^{29}$ J.-X. Zhu, K. O. Rasmussen, A. R. Bishop, and A. V. Balatsky, arXiv:cond-mat/0407215.

${ }^{30}$ G. Teobaldi, M. Peñalba, A. Arnau, N. Lorente, and W. A. Hofer, Phys. Rev. B 76, 235407 (2007).

${ }^{31}$ J. I. Cerdá, M. A. Van Hove, P. Sautet, and M. Salmerón, Phys. Rev. B 56, 15885 (1997); see also http://www.icmm.csic.es/jcerda/

${ }^{32}$ B. Janta-Polczynski, J. I. Cerdá, G. Ethier-Majcher, K. Piyakis, and A. Rochefort, J. Appl. Phys. 104, 23702 (2008).

${ }^{33}$ J. M. Soler, E. Artacho, J. D. Gale, A. García, J. Junquera, P. Ordejón, and D. Sánchez-Portal, J. Phys.: Condens. Matter 14, 2745 (2002).

${ }^{34}$ M. Paulsson, T. Frederiksen, H. Ueba, N. Lorente, and M. Brandbyge, Phys. Rev. Lett. 100, 226604 (2008).

${ }^{35}$ A. Garcia-Lekue, D. Sánchez-Portal, A. Arnau, and T. Frederiksen, Phys. Rev. B 83, 155417 (2011).

${ }^{36}$ S. Corbel, J. Cerdá, and P. Sautet, Phys. Rev. B 60, 1989 (1999).

${ }^{37}$ C. Rogero, J. A. Martin-Gago, and J. I. Cerdá, Phys. Rev. B 74, 121404 (2006).

${ }^{38}$ L. Kleinman and D. M. Bylander, Phys. Rev. Lett. 48, 1425 (1982). 
${ }^{39}$ M. Büttiker, Y. Imry, R. Landauer, and S. Pinhas, Phys. Rev. B 31, 6207 (1985).

${ }^{40}$ G. Meyer, L. Bartels, and K. H. Rieder, Superlattices Microstruct. 25, 463 (1999).

${ }^{41}$ D. Sánchez-Portal, E. Artacho, and J. M. Soler, J. Phys.: Condens. Matter 8, 3859 (1996).

${ }^{42}$ S. García-Gil, A. García, Nicolás Lorente, and P. Ordejón, Phys. Rev. B 79, 075441 (2009).
${ }^{43}$ J. P. Perdew, K. Burke, and M. Ernzerhof, Phys. Rev. Lett. 77, 3865 (1996).

${ }^{44}$ T. Frederiksen, N. Lorente, M. Paulsson, and M. Brandbyge, Phys. Rev. B 75, 235441 (2007).

${ }^{45}$ N. Lorente, M. Persson, L. J. Lauhon, and W. Ho, Phys. Rev. Lett. 86, 2593 (2001).

${ }^{46}$ R. Cuadrado, J. I. Cerdá, Y. Wang, G. Xin, R. Berndt, and H. Tang, J. Chem. Phys. 133, 154701 (2010). 